\title{
Complex Bosonic Many-Body Models: Overview of the Small Field Parabolic Flow
}

\author{
Tadeusz Balaban, Joel Feldman, Horst Knörrer and \\ Eugene Trubowitz
}

\begin{abstract}
This paper is a contribution to a program to see symmetry breaking in a weakly interacting many boson system on a three-dimensional lattice at low temperature. It provides an overview of the analysis, given in Balaban et al. (The small field parabolic flow for bosonic manybody models: part 1-main results and algebra, arXiv:1609.01745, 2016, The small field parabolic flow for bosonic many-body models: part 2 fluctuation integral and renormalization, arXiv:1609.01746, 2016), of the 'small field' approximation to the 'parabolic flow' which exhibits the formation of a 'Mexican hat' potential well.
\end{abstract}

It is our long-term goal to rigorously demonstrate symmetry breaking in a gas of bosons hopping on a three-dimensional lattice. Technically, to show that the correlation functions decay at a nonintegrable rate when the chemical potential is sufficiently positive, the nonintegrability reflecting the presence of a long range Goldstone boson mediating the interaction between quasiparticles in the superfluid condensate. It is already known $[19,20]$ that the correlation functions are exponentially decreasing when the chemical potential is sufficiently negative. See, for example, [22] and [30, §19] for an introduction to symmetry breaking in general, and $[1,18,23,28]$ as general references to Bose-Einstein condensation. See $[17,21,26,29]$ for other mathematically rigorous work on the subject.

We start with a brief, formula free, summary of the program and its current state. Then we'll provide a more precise, but still simplified, discussion of the portion of the program that controls the small field parabolic flow.

Research supported in part by the Natural Sciences and Engineering Research Council of Canada and the Forschungsinstitut für Mathematik, ETH Zürich. 
The program was initiated in $[3,4]$, where we expressed the positive temperature partition function and thermodynamic correlation functions in a periodic box (a discrete three-dimensional torus) as 'temporal' ultraviolet limits of four-dimensional (coherent state) lattice functional integrals (see also [27]). By a lattice functional integral, we mean an integral with one (in this case complex) integration variable for each point of the lattice. By a 'temporal' ultraviolet limit, we mean a limit in which the lattice spacing in the inverse temperature direction (imaginary time direction) is sent to zero while the lattice spacing in the three spatial directions is held fixed.

In [7], ${ }^{1}$ by a complete large field/small field renormalization group analysis, we expressed the temporal ultraviolet limit for the partition function, ${ }^{2}$ still in a periodic box, as a four-dimensional lattice functional integral with the lattice spacing in all four directions being of the order one, preparing the way for an infrared renormalization group analysis of the thermodynamic limit.

This overview concerns the next stage of the program, which is contained in $[13,14]$ and the supporting papers $[9-12,15,16]$. There we initiate the infrared analysis by tracking, in the small field region, the evolution of the effective interaction generated by the iteration of a renormalization group map that is taylored to a parabolic covariance ${ }^{3}$ : in each renormalization group step the spatial lattice directions expand by a factor ${ }^{4} L>1$, the inverse temperature direction expands by a factor $L^{2}$ and the running chemical potential grows by a factor of $L^{2}$, while the running coupling constant decreases by a factor of $L^{-1}$. Consequently, the effective potential, initially close to a paraboloid, develops into a Mexican hat with a moderately large radius and a moderately deep circular well of minima. [13,14] ends after a finite number (of the order of the magnitude of the logarithm of the coupling constant) of steps once the chemical potential, which initially was of the order of the coupling constant, has grown to a small ' $\epsilon$ ' power of the coupling constant. Then we can no longer base our analysis on expansions about zero field, because the renormalization group iterations have moved the effective model away from the trivial noninteracting fixed point.

In the next stage of the construction, we plan to continue the parabolic evolution in the small field regime, but expanding around fields concentrated at the bottom of the (Mexican hat shaped) potential well rather around zero (much as is done in the Bogoliubov Ansatz) and track it through an additional finite number of steps until the running chemical potential is sufficiently larger than one. At that point, we will turn to a renormalization group map with a scaling taylored to an elliptic covariance that expands both the temporal (inverse temperature) and spatial lattice directions by the same factor $L$. It is expected that the elliptic evolution can be controlled through infinitely many steps, all the way to the symmetry broken fixed point. The system is

\footnotetext{
${ }^{1}$ See also [8] for a more pedagogical introduction.

2 A similar analysis will yield the corresponding representations for the correlation functions.

${ }^{3}$ Morally, the $1+3$-dimensional heat operator.

${ }^{4} L$ is a fixed, sufficiently large, odd natural number.
} 
superrenormalizable in the entire parabolic regime because the running coupling constant is geometrically decreasing. However in the elliptic regime, the system is only strictly renormalizable.

The final stage(s) of the program concern the control of the large field contributions in both the parabolic and elliptic regimes.

The technical implementation of the parabolic renormalization group in $[13,14]$ proceeds much as in $[6,7]$, except that we are restricting our attention to the small field regime and

○ we use $1+3$-dimensional block spin averages, as in $[2,24,25]$. In [7], we had used decimation, which was suited to the effectively one-dimensional problem of evaluating the temporal ultraviolet limit.

- Otherwise, the stationary phase calculation that controls oscillations is similar, but technically more elaborate.

- The essential complication is that the critical fields and background fields are now solutions to (weakly) nonlinear systems of parabolic equations.

- The Stokes' argument that allows us to shift the multidimensional integration contour to the 'reals' and

o the evaluation of the fluctuation integrals is similar.

- However, there is an important new feature: The chemical potential has to be renormalized.

To analyze the output of the block spin convolution (a single renormalization group step), it is de rigueur for the small field/large field style of renormalization group implementations to introduce local small field conditions on the integrand and then decompose the integral into the sum over all partitions of the discrete torus into small and large field regions on which the conditions are satisfied and violated, respectively. Small field contributions are to be controlled by powers of the coupling constant $\mathfrak{v}_{0}$ (a suitable norm of the two body interaction) uniformly in the volume of the small field region. Large field contributions are to be controlled by a factor $e^{-1 / \mathfrak{v}_{0}^{\varepsilon}}, \varepsilon>0$, raised to the volume of the large field region. Morally, in small field regions, perturbation expansions in the coupling constant converge and exhibit all physical phenomena. Large field regions give multiplicative corrections that are smaller than any power of the coupling constant. So, in the leading terms, every point is small field.

If the actions in our functional integrals were sums of positive terms (as in a Euclidean $\mathrm{O}(\mathrm{n})$ model), it would be routine to extract an exponentially small factor per point of a large field region. They are not. There are explicit purely imaginary terms. In $[13,14]$, we analyze the parabolic flow of the leading term, in which all points are small field, as long as it is possible to expand around zero field. Nevertheless, we show (see, [15]) that our actions do have positivity properties and consequently there is at least one factor $e^{-1 / \mathfrak{v}_{0}^{\varepsilon}}$ whenever there is a large field region. A stronger bound of a factor per point of a large field region is reasonable and would be the main ingredient for controlling the full parabolic renormalization group flow in this regime. 
We now formally introduce the main objects of discussion and enough machinery to allow technical (but simplified) statements of the main results of $[13,14]$ and the methods used to establish them.

One conclusion of our previous work in [7] is that the purely small field contribution to the partition function for a gas of bosons hopping on a threedimensional discrete torus $X=\mathbb{Z}^{3} / L_{\mathrm{sp}} \mathbb{Z}^{3}$ (where $L_{\mathrm{sp}}$, a power of $L$, is the spatial infrared regulator which will ultimately be sent to infinity) takes the form

$$
\int_{S_{0}} \prod_{x \in \mathcal{X}_{0}} \frac{d \psi(x)^{*} \wedge d \psi(x)}{2 \pi \imath} e^{\mathcal{A}_{0}\left(\psi^{*}, \psi\right)}
$$

where

- $\mathcal{X}_{0}=\mathbb{Z} / L_{\mathrm{tp}} \mathbb{Z} \times X$ is a $1+3$-dimensional discrete torus with points $x=\left(x_{0}, \mathbf{x}\right)$. Here, $L_{\mathrm{tp}} \approx \frac{1}{k T}$, also a power of $L$, is the inverse temperature infrared regulator, which can ultimately be sent to infinity to get the temperature zero limit.

- $\psi \in \mathbb{C}^{\mathcal{X}_{0}}$ is a complex valued field on $\mathcal{X}_{0}, \psi^{*}$ is the complex conjugate field and, for each $x \in \mathcal{X}_{0}, \frac{d \psi(x)^{*} \wedge d \psi(x)}{2 \imath}$ is the standard Lebesgue measure on $\mathbb{C}$.

- $S_{0}=\left\{\psi \in \mathbb{C}^{\mathcal{X}_{0}}|| \psi(x)\left|\leq \mathfrak{v}_{0}^{-1 / 3+\epsilon},\right| \partial_{\nu} \psi(x) \mid \leq \mathfrak{v}_{0}^{-1 / 3+\epsilon}, \nu=0,1,2,3\right.$, $\left.x \in \mathcal{X}_{0}\right\}$

where the small 'coupling constant' $\mathfrak{v}_{0}$ is an exponentially, tree length weighted $L^{1}-L^{\infty}$-norm (see the discussion of norms at the end of this overview or [13, Definition 1.9]) of an effective interaction $V_{0}$ (see [13, Proposition D.1]). Here, $\partial_{\nu}, \nu=0,1,2,3$, is the forward difference operator in the $x_{\nu}$ direction.

- Let $\psi_{*}$ be another arbitrary element of $\mathbb{C}^{\mathcal{X}_{0}}$. ( $\psi_{*}$ is not to be confused with the complex conjugate $\psi^{*}$ of $\psi$.)

- $\mathcal{A}_{0}\left(\psi_{*}, \psi\right)=-A_{0}\left(\psi_{*}, \psi\right)+p_{0}\left(\psi_{*}, \psi, \nabla \psi_{*}, \nabla \psi\right)$. The action $\mathcal{A}_{0}\left(\psi^{*}, \psi\right)$ determining the partition function is the restriction

$$
\mathcal{A}_{0}\left(\psi^{*}, \psi\right)=\left.\mathcal{A}_{0}\left(\psi_{*}, \psi\right)\right|_{\psi_{*}=\psi^{*}}
$$

of $\mathcal{A}_{0}\left(\psi_{*}, \psi\right)$ to the 'real' subspace $\psi_{*}=\psi^{*}$ of $\mathbb{C}^{\mathcal{X}_{0}} \times \mathbb{C}^{\mathcal{X}_{0}}$. Here, $\nabla$ is the (four-dimensional) discrete gradient operator.

- 'Morally,' $A_{0}\left(\psi_{*}, \psi\right)=\left\langle\psi_{*},\left(-\partial_{0}+h\right) \psi\right\rangle_{0}+\mathcal{V}_{0}\left(\psi_{*}, \psi\right)-\mu_{0}\left\langle\psi_{*}, \psi\right\rangle_{0}$, where

$\circ\langle f, g\rangle_{0}=\sum_{x \in \mathcal{X}_{0}} f(x) g(x)$ is the natural real inner product on $\mathbb{C}^{\mathcal{X}_{0}}$

$\circ \quad h$ is a nonnegative, second-order, elliptic (lattice) pseudodifferential operator acting on $X$-for example, a constant times minus the spatial discrete Laplacian

○ $\mathcal{V}_{0}\left(\psi_{*}, \psi\right)=\frac{1}{2} \sum_{\mathcal{X}_{0}^{4}} V_{0}\left(x_{1}, x_{2}, x_{3}, x_{4}\right) \psi_{*}\left(x_{1}\right) \psi\left(x_{2}\right) \psi_{*}\left(x_{3}\right) \psi\left(x_{4}\right)$ is a quartic monomial whose kernel $V_{0}$ is translation invariant with

$$
\sum_{\mathcal{X}_{0}^{3}} V_{0}\left(0, x_{2}, x_{3}, x_{4}\right)>0
$$


- $\mu_{0}$ is essentially the chemical potential.

- Let $\psi_{* \nu}, \psi_{\nu}, \nu=0,1,2,3$, be the names of new arbitrary elements of $\mathbb{C}^{\mathcal{X}_{0}}$. The perturbative correction $p_{0}\left(\psi_{*}, \psi,\left\{\psi_{* \nu}\right\}_{\nu=0}^{3},\left\{\psi_{\nu}\right\}_{\nu=0}^{3}\right)$, to the principal contribution $-A_{0}$, in $\mathcal{A}_{0}$, is a power series in the ten variables $\psi_{*}, \psi,\left\{\psi_{* \nu}, \psi_{\nu}\right\}_{\nu=0}^{3}$, with no $\psi_{*}(x) \psi(y)$ terms, such that each nonzero term has as many factors with asterisks as factors without asterisks. That is, $p_{0}$ conserves particle number. It converges on

$$
\begin{aligned}
\left\{\left(\psi_{*}, \psi,\left\{\psi_{* \nu}, \psi_{\nu}\right\}_{\nu=0}^{3}\right) \in \mathbb{C}^{10 \mathcal{X}_{0}}|| \psi_{(*)}(x)|,| \psi_{(*) \nu}(x) \mid\right. & \leq \mathfrak{v}_{0}^{-1 / 3+\varepsilon}, \\
& \left.0 \leq \nu \leq 3, x \in \mathcal{X}_{0}\right\}
\end{aligned}
$$

where ' $(*)$ ' means 'either with $*$ or without $*$.'

See [13, Proposition D.1] for more details.

For convenience, set

$$
F_{0}\left(\psi^{*}, \psi\right)=e^{\mathcal{A}_{0}\left(\psi^{*}, \psi\right)} \chi_{S_{0}}(\psi) .
$$

With this notation, the partition function is

$$
\int \prod_{x \in \mathcal{X}_{0}} \frac{d \psi(x)^{*} \wedge d \psi(x)}{2 \pi \imath} F_{0}\left(\psi^{*}, \psi\right)+O\left(e^{-1 / \mathfrak{v}_{0}^{\varepsilon}}\right) .
$$

It is natural to study the partition function using a steepest descent or stationary phase analysis. The exponential $e^{\left\langle\psi^{*}, \partial_{0} \psi\right\rangle}$ is purely oscillatory because the quadratic form $\left\langle\psi^{*}, \partial_{0} \psi\right\rangle$ is pure imaginary. Fortunately, our partition function, $\mathcal{Z}$, has the essential feature that there is an analytic function $\mathcal{A}_{0}\left(\psi_{*}, \psi\right)$ on a neighborhood of the origin in $\mathbb{C}^{\mathcal{X}_{0}} \times \mathbb{C}^{\mathcal{X}_{0}}$ whose restriction to the real subspace is the 'small field' action. Our renormalization group analysis of the oscillating integral defining $\mathcal{Z}$ is based on the critical points of $A_{0}\left(\psi_{*}, \psi\right)=\left\langle\psi_{*},\left(-\partial_{0}+h\right) \psi\right\rangle+\mathcal{V}_{0}\left(\psi_{*}, \psi\right)-\mu_{0}\left\langle\psi_{*}, \psi\right\rangle$ in $\mathbb{C}^{\mathcal{X}_{0}} \times \mathbb{C}^{\mathcal{X}_{0}}$ that typically do not lie in the real subspace, and a multidimensional Stokes' contour shifting construction that is only possible because $p_{0}\left(\psi_{*}, \psi\right)$ is analytic.

We now formally introduce the 'block spin' renormalization group transformations that are used in this paper. Let $\mathcal{X}_{-1}$ be the subgroup $L^{2} \mathbb{Z} / L_{\mathrm{tp}} \mathbb{Z} \times$ $L \mathbb{Z}^{3} / L_{\mathrm{sp}} \mathbb{Z}^{3}$ of $\mathcal{X}_{0}$. Observe that the distance between points of $\mathcal{X}_{-1}$ on the inverse temperature axis is $L^{2}$ and on the spatial axes is $L$, and that $\left|\mathcal{X}_{-1}\right|=L^{-5}\left|\mathcal{X}_{0}\right|$. Also, let $\mathcal{Q}^{(0)}: \mathbb{C}^{\mathcal{X}_{0}} \rightarrow \mathbb{C}^{\mathcal{X}_{-1}}$ be a linear operator that commutes with complex conjugation. We will make a specific choice of $\mathcal{Q}^{(0)}$ later. It will be a 'block spin averaging' operator with, for each $y \in \mathcal{X}_{-1}$, $\left(\mathcal{Q}^{(0)} \psi\right)(y)$ being 'morally' the average value of $\psi$ in the $L^{2} \times L \times L \times L$ block centered on $y$. Insert into the integral of (2)

$$
1=\frac{1}{N^{(0)}} \int_{\mathbb{C}^{\mathcal{X}}-1} \prod_{y \in \mathcal{X}_{-1}} \frac{d \theta(y)^{*} \wedge d \theta(y)}{2 \pi \imath} e^{-\frac{1}{L^{2}}\left\langle\theta_{*}-\mathcal{Q}^{(0)} \psi^{*}, \theta-\mathcal{Q}^{(0)} \psi\right\rangle_{-1}}
$$

where $\langle f, g\rangle_{-1}=L^{5} \sum_{y \in \mathcal{X}_{-1}} f(y) g(y)$ is the natural real inner product on $\mathbb{C}^{\mathcal{X}_{-1}}$ and $N^{(0)}$ is a normalization constant. Then exchange the order of the 
$\psi$ and $\theta$ integrals. This gives

$$
\int \prod_{x \in \mathcal{X}_{0}} \frac{d \psi(x)^{*} \wedge d \psi(x)}{2 \pi \imath} F_{0}\left(\psi^{*}, \psi\right)=\int \prod_{y \in \mathcal{X}_{-1}} \frac{d \theta(y)^{*} \wedge d \theta(y)}{2 \pi \imath} B_{1}\left(\theta_{*}, \theta\right)
$$

where, by definition, the block spin transform of $F_{0}\left(\psi_{*}, \psi\right)$ associated with $\mathcal{Q}^{(0)}$ with external fields $\theta$ and $\theta_{*}$ is

$B_{1}\left(\theta_{*}, \theta\right)=\frac{1}{N^{(0)}} \int_{\mathbb{C}^{\mathcal{X}_{0}}} \prod_{x \in \mathcal{X}_{0}} \frac{d \psi(x)^{*} \wedge d \psi(x)}{2 \pi \imath} e^{-\frac{1}{L^{2}}\left\langle\theta_{*}-\mathcal{Q}^{(0)} \psi^{*}, \theta-\mathcal{Q}^{(0)} \psi\right\rangle_{-1}} F_{0}\left(\psi^{*}, \psi\right)$

Here $\theta, \theta_{*}$ are two arbitrary elements of $\mathbb{C}^{\mathcal{X}_{-1}}$.

It can be awkward to compare functions defined on discrete tori with different lattice spacings. So, we scale $\mathcal{X}_{-1}$ down to the unit discrete torus

$$
\mathcal{X}_{0}^{(1)}=\mathbb{Z} / \frac{L_{\mathrm{tp}}}{L^{2}} \mathbb{Z} \times \mathbb{Z}^{3} / \frac{L_{\mathrm{sp}}}{L} \mathbb{Z}^{3}
$$

using the 'parabolic' scaling map $x \in \mathcal{X}_{0}^{(1)} \rightarrow\left(L^{2} x_{0}, L \mathbf{x}\right) \in \mathcal{X}_{-1}$, which is an isomorphism of Abelian groups. Abusing notation, we consciously use the symbol $\psi(x)$ as the name of a field on the unit torus $\mathcal{X}_{0}^{(1)}$ even though it was used before as the name of a field on the unit torus $\mathcal{X}_{0}$. By definition, the block spin renormalization group transform of $F_{0}\left(\psi^{*}, \psi\right)$ associated with $\mathcal{Q}^{(0)}$ with external fields $\psi$ and $\psi_{*}$ in $\mathbb{C}^{\mathcal{X}_{0}^{(1)}}$ is

$$
F_{1}\left(\psi_{*}, \psi\right)=B_{1}\left(\mathbb{S}^{-1} \psi_{*}, \mathbb{S}^{-1} \psi\right) \quad \text { where } \quad\left(\mathbb{S}^{-1} \psi\right)\left(y_{0}, \mathbf{y}\right)=L^{-3 / 2} \psi\left(\frac{y_{0}}{L^{2}}, \frac{\mathbf{y}}{L}\right)(3)
$$

for any $\psi \in \mathbb{C}^{\mathcal{X}_{0}^{(1)}}$. The 'parabolic' exponent $-3 / 2$ has been chosen so that ${ }^{5}$ $\left\langle\mathbb{S} \theta^{*},\left(\partial_{0}+\Delta\right) \mathbb{S} \theta\right\rangle_{0}=\left\langle\theta^{*},\left(\partial_{0}+\Delta\right) \theta\right\rangle_{-1}$. We now have

$$
\begin{gathered}
L^{-3\left|\mathcal{X}_{0}^{(1)}\right|} \int_{\mathbb{C}^{\mathcal{X}_{0}^{(1)}}} \prod_{x \in \mathcal{X}_{0}^{(1)}} \frac{d \psi(x)^{*} \wedge d \psi(x)}{2 \pi \imath} F_{1}\left(\psi^{*}, \psi\right) \\
=\int_{\mathbb{C}^{\mathcal{X}_{0}}} \prod_{x \in \mathcal{X}_{0}} \frac{d \psi(x)^{*} \wedge d \psi(x)}{2 \pi \imath} F_{0}\left(\psi^{*}, \psi\right)
\end{gathered}
$$

the original small field part of the partition function.

Repeat the construction.

○ Let $\mathcal{X}_{-1}^{(2)}$ be the subgroup $L^{2} \mathbb{Z} / \frac{L_{\mathrm{tp}}}{L^{2}} \mathbb{Z} \times L \mathbb{Z}^{3} / \frac{L_{\mathrm{sp}}}{L} \mathbb{Z}^{3}$ of $\mathcal{X}_{0}^{(1)}$ and

○ let $\mathcal{Q}^{(1)}: \mathbb{C}^{\mathcal{X}_{0}^{(1)}} \rightarrow \mathbb{C}^{\mathcal{X}_{-1}^{(2)}}$ be a linear 'block averaging' operator that commutes with complex conjugation.

○ Introduce the unit discrete torus $\mathcal{X}_{0}^{(2)}=\mathbb{Z} / \frac{L_{\mathrm{tp}}}{L^{4}} \mathbb{Z} \times \mathbb{Z}^{3} / \frac{L_{\mathrm{sp}}}{L^{2}} \mathbb{Z}^{3}$ and

○ the isomorphism $x=\left(x_{0}, \mathbf{x}\right) \in \mathcal{X}_{0}^{(2)} \rightarrow\left(L^{2} x_{0}, L \mathbf{x}\right) \in \mathcal{X}_{-1}^{(2)}$.

As before, integrate against the normalized Gaussian to obtain the block spin transform of $F_{1}$ associated with $\mathcal{Q}^{(1)}$

$B_{2}\left(\theta_{*}, \theta\right)=\frac{1}{N^{(1)}} \int_{\mathbb{C}^{\mathcal{X}_{0}^{(1)}}} \prod_{x \in \mathcal{X}_{0}^{(1)}} \frac{d \psi(x)^{*} \wedge d \psi(x)}{2 \pi \imath} e^{-\frac{1}{L^{2}}\left\langle\theta_{*}-\mathcal{Q}^{(1)} \psi^{*}, \theta-\mathcal{Q}^{(1)} \psi\right\rangle_{-1}} F_{1}\left(\psi^{*}, \psi\right)$

\footnotetext{
${ }^{5} \operatorname{In}\left\langle\theta^{*},\left(\partial_{0}+\Delta\right) \theta\right\rangle_{-1}, \partial_{0}$ is the forward difference operator on $\mathcal{X}_{-1}$. That is, $\left(\partial_{0} f\right)(y)=$ $\frac{f\left(y_{0}+L^{2}, \mathbf{y}\right)-f\left(y_{0}, \mathbf{y}\right)}{L^{2}}$. Similarly, for spatial difference operators.
} 
and then rescale to obtain the block spin renormalization group transform

$$
F_{2}\left(\psi_{*}, \psi\right)=B_{2}\left(\mathbb{S}^{-1} \psi_{*}, \mathbb{S}^{-1} \psi\right)
$$

where $\left(\mathbb{S}^{-1} \psi\right)\left(y_{0}, \mathbf{y}\right)=L^{-3 / 2} \psi\left(\frac{y_{0}}{L^{2}}, \frac{\mathbf{y}}{L}\right)$ for any $\psi \in \mathbb{C}^{\mathcal{X}_{0}^{(2)}}$. Interchanging the order of integration,

$$
\begin{aligned}
& L^{-3\left|\mathcal{X}_{0}^{(2)}\right|} L^{-3\left|\mathcal{X}_{0}^{(1)}\right|} \int_{\mathbb{C}^{\mathcal{X}_{0}^{(2)}}} \prod_{x \in \mathcal{X}_{0}^{(2)}} \frac{d \psi(x)^{*} \wedge d \psi(x)}{2 \pi \imath} F_{2}\left(\psi^{*}, \psi\right) \\
& =\int_{\mathbb{C}^{\mathcal{X}_{0}}} \prod_{x \in \mathcal{X}_{0}} \frac{d \psi(x)^{*} \wedge d \psi(x)}{2 \pi \imath} F_{0}\left(\psi^{*}, \psi\right) .
\end{aligned}
$$

We keep repeating the construction to generate a sequence $F_{n}\left(\psi_{*}, \psi\right), n \geq 1$, of functions defined on spaces $\mathbb{C}^{\mathcal{X}_{0}^{(n)}} \times \mathbb{C}^{\mathcal{X}_{0}^{(n)}}$. Balaban et al. [13,14] concern a sequence $F_{n}^{(S F)}\left(\psi_{*}, \psi\right)$ of 'small field' approximations to the $F_{n}$ 's. We expect and provide some supporting motivation for, but do not prove, that $F_{n}=$ $F_{n}^{(S F)}+O\left(e^{-1 / \mathfrak{v}_{0}^{\varepsilon}}\right)$. For the precise definition, see $[13, \S 1.2$ and, in particular, Definition 1.6]. For the supporting motivation see [15].

To make a specific choice for the, to this point arbitrary, sequence $\mathcal{Q}^{(0)}$, $\ldots, \mathcal{Q}^{(n)}, \ldots$ of block averaging operators, let $q(x)$ be a nonnegative, compactly supported, even function on $\mathbb{Z} \times \mathbb{Z}^{3}$ and $Q$ the associated convolution operator $^{6}$

$$
(Q \psi)(y)=\sum_{x \in \mathbb{Z} \times \mathbb{Z}^{3}} q(x) \psi(y+[x]), \quad \psi \in \mathbb{C}^{\mathcal{X}_{0}^{(n)}}, y \in \mathcal{X}_{-1}^{(n+1)} \subset \mathcal{X}_{0}^{(n)}
$$

where $[x]$ is the point in the quotient $\mathcal{X}_{0}^{(n)}=\mathbb{Z} / \frac{L_{\mathrm{tp}}}{L^{2 n}} \mathbb{Z} \times \mathbb{Z}^{3} / \frac{L_{\mathrm{sp}}}{L^{n}} \mathbb{Z}^{3}$ represented by $x \in \mathbb{Z} \times \mathbb{Z}^{3}$. By construction, $Q \psi \in \mathbb{C}^{\mathcal{X}_{-1}^{(n+1)}}$. We fix $q(x)$ to be the convolution of the indicator function of the (discrete) rectangle $\left[-\frac{L^{2}-1}{2}, \frac{L^{2}-1}{2}\right] \times\left[-\frac{L-1}{2}, \frac{L-1}{2}\right]^{3}$ in $\mathbb{Z} \times \mathbb{Z}^{3}$ convolved with itself four times and normalized so that its sum over $\mathbb{Z} \times \mathbb{Z}^{3}$ is one. In $[13,14]$, the basic objects are the 'small field' block spin renormalization iterates $F_{n}^{(S F)}\left(\psi_{*}, \psi\right)$, where at each step $Q$ is chosen to be convolution with the fixed kernel $q$.

If we had defined $Q$ by convolving just with the indicator function of the rectangle itself, properly normalized, then $(Q \psi)(y)$ would be the usual average of $\psi(x)$ over the rectangular box in $\mathcal{X}_{0}^{(n)}$ centered at $y$ with sides $L^{2}$ and $L$. We work with the smoothed averaging kernel rather than the sharp one for technical reasons: Commutators $\left[\partial_{\nu}, Q\right]$ are routinely generated and are small enough when $Q$ is smooth enough. For the rest of this overview, we will pretend that $q$ is just the indicator function of the rectangle and formulate our results as if this were the case. We will also pretend that the operator $h$ on $X$ appearing in the action $A_{0}\left(\psi_{*}, \psi\right)$ is (minus) the lattice Laplacian. Full, technically complete, statements are in $[13, \S 1.6]$.

\footnotetext{
${ }^{6}$ By abuse of notation, we use the same symbol $Q$ for the convolution operator acting on all of the spaces $\mathbb{C}^{\mathcal{X}_{0}^{(n)}}$.
} 
Our main result is: If $\epsilon>0$ and $\mathfrak{v}_{0}$ are small enough and $L$ is large enough, there exists $a^{7} \mu_{*}=O\left(\mathfrak{v}_{0}\right)$, such that for all ${ }^{8} \mu_{*}+\mathfrak{v}_{0}^{5 / 4}<\mu_{0}<\mathfrak{v}_{0}^{9 / 10}$ and all $n<\frac{2}{5} \frac{\log 1 \mathfrak{o}_{0}}{\log L}$, the 'small field approximations' $F_{n}^{(S F)}$ to the $F_{n}$ 's are

$$
\begin{aligned}
F_{n}^{(S F)}\left(\psi_{*}, \psi\right)= & \frac{1}{\mathcal{Z}_{n}} \exp \left\{-A_{n}\left(\psi_{*}, \psi, \phi_{* n}\left(\psi_{*}, \psi\right), \phi_{n}\left(\psi_{*}, \psi\right)\right)\right. \\
& \left.+p_{n}\left(\psi_{*}, \psi, \nabla \psi_{*}, \nabla \psi\right)\right\} \\
A_{n}= & a_{n}\left\langle\left(\psi_{*}-Q_{n} \phi_{* n}\right),\left(\psi-Q_{n} \phi_{n}\right)\right\rangle_{0}+\left\langle\phi_{* n},\left(-\partial_{0}-\Delta\right) \phi_{n}\right\rangle_{n} \\
& -\mu_{n}\left\langle\phi_{* n}, \phi_{n}\right\rangle_{n}+\mathcal{V}_{n}\left(\phi_{* n}, \phi_{n}\right)
\end{aligned}
$$

on the domain

$$
\begin{gathered}
S_{n}=\left\{\left(\psi_{*}, \psi\right) \in \mathbb{C}^{2 \mathcal{X}_{0}^{(n)}}|| \psi_{(*)}(x)\left|\leq \kappa_{n},\right| \partial_{\nu} \psi_{(*)}(x) \mid \leq \kappa_{n}^{\prime},\right. \\
\left.0 \leq \nu \leq 3, x \in \mathcal{X}_{0}^{(n)}\right\}
\end{gathered}
$$

and zero on its complement. Here,

- you can think of the radii $\kappa_{n}$ and $\kappa_{n}^{\prime}$ as being roughly $L^{\frac{3}{4} n_{\mathfrak{v}_{0}}}{ }^{-\frac{1}{3}+\epsilon}$ and $L^{\frac{3}{8} n_{\mathfrak{v}_{0}}}{ }^{-\frac{1}{3}+\epsilon}$, respectively. Explicit expressions for $\kappa_{n}$ and $\kappa_{n}^{\prime}$ are given in [13, Definition 1.11.a].

- $\phi_{* n}\left(\psi_{*}, \psi\right)$ and $\phi_{n}\left(\psi_{*}, \psi\right)$ are (nonlinear) maps from an open neighborhood of the origin in $\mathbb{C}^{\mathcal{X}_{0}^{(n)}} \times \mathbb{C}^{\mathcal{X}_{0}^{(n)}}$ to $\mathbb{C}^{\mathcal{X}_{n}}$, where $\mathcal{X}_{n}$ is the discrete torus, isomorphic to $\mathcal{X}_{0}$, but scaled down to have lattice spacing $L^{-2 n}$ in the time direction and $L^{-n}$ in the spatial directions. ${ }^{9}$ We say more about them in the last of this sequence of bullets. Given 'external fields' $\psi_{*}, \psi$, the functions $\phi_{* n}\left(\psi_{*}, \psi\right)(u), \phi_{n}\left(\psi_{*}, \psi\right)(u)$ on $\mathcal{X}_{n}$ are referred to as the 'background fields' at scale $n$.

- $\langle f, g\rangle_{0}=\sum_{x \in \mathcal{X}_{0}^{(n)}} f(x) g(x)$ and $\langle f, g\rangle_{n}=L^{-5 n} \sum_{u \in \mathcal{X}_{n}} f(u) g(u)$ are the natural real inner products on $\mathbb{C}^{\mathcal{X}_{0}^{(n)}}$ and $\mathbb{C}^{\mathcal{X}_{n}}$.

- $Q_{n}: \mathbb{C}^{\mathcal{X}_{n}} \rightarrow \mathbb{C}^{\mathcal{X}_{0}^{(n)}}$ is the linear map for which $\left(Q_{n} f\right)(x)$ is the average of $f \in \mathbb{C}^{\mathcal{X}_{n}}$ over the square box in $\mathcal{X}_{n}$ centered at $x \in \mathcal{X}_{0}^{(n)}$ with sides

1. (This box contains $L^{2 n} \times\left(L^{n}\right)^{3}$ points of $\mathcal{X}_{n}$.)

- $a_{n}=\frac{1-L^{-2}}{1-L^{-2 n}}$

- $-\partial_{0}-\Delta$ is the natural heat operator on the 'fine' discrete torus $\mathcal{X}_{n}$.

- For each $f_{*}, f \in \mathbb{C}^{\mathcal{X}_{n}}$

$$
\mathcal{V}_{n}\left(f_{*}, f\right)=\frac{1}{2}\left(\frac{1}{L^{5 n}}\right)^{4} \sum_{\substack{u_{j} \in \mathcal{X}_{n} \\ j=1,2,3,4}} V_{n}\left(u_{1}, u_{2}, u_{3}, u_{4}\right) f_{*}\left(u_{1}\right) f\left(u_{2}\right) f_{*}\left(u_{3}\right) f\left(u_{4}\right)
$$

where $V_{n}\left(u_{1}, u_{2}, u_{3}, u_{4}\right)$ is close to

$$
V_{n}^{(u)}\left(u_{1}, u_{2}, u_{3}, u_{4}\right)=\frac{1}{L^{n}}\left(L^{5 n}\right)^{3} V_{0}\left(U_{1}, U_{2}, U_{3}, U_{4}\right), \quad U_{j}=\left(L^{2 n} u_{j 0}, L^{n} \mathbf{u}_{j}\right)(4)
$$

\footnotetext{
${ }^{7}$ An explicit formula for $\mu_{*}$ is given in [13, (1.19)].

${ }^{8}$ We are weakening some of the statements, for pedagogical reasons. In particular, the sets of allowed $\mu_{0}$ 's and $n$ 's are a bit larger than the sets specified here.

$9 \mathcal{X}_{n}=\frac{1}{L^{2 n}} \mathbb{Z} / \frac{L_{\mathrm{tp}}}{L^{2 n}} \mathbb{Z} \times \frac{1}{L^{n}} \mathbb{Z}^{3} / \frac{L_{\mathrm{sp}}}{L^{n}} \mathbb{Z}^{3}$ and the map $u \in \mathcal{X}_{n} \mapsto x=\left(L^{2 n} u_{0}, L^{n} \mathbf{u}\right) \in \mathcal{X}_{0}$ is an isomorphism of Abelian groups.
} 
- The perturbative correction $p_{n}\left(\psi_{*}, \psi,\left\{\psi_{* \nu}\right\}_{\nu=0}^{3},\left\{\psi_{\nu}\right\}_{\nu=0}^{3}\right)$ is a power series in the ten variables $\psi_{*}, \psi,\left\{\psi_{* \nu}, \psi_{\nu}\right\}_{\nu=0}^{3} \in \mathbb{C}^{\mathcal{X}_{0}^{(n)}}$, with no $\psi_{*}(x) \psi(y)$ or constant terms, such that each nonzero term has as many factors with asterisks as factors without asterisks. It converges ${ }^{10}$ when $\left|\psi_{(*)}(x)\right| \leq \kappa_{n}$ and $\left|\psi_{(*) \nu}(x)\right| \leq \kappa_{n}^{\prime}$ for all $0 \leq \nu \leq 3$ and $x \in \mathcal{X}_{0}^{(n)}$.

- $\mathcal{Z}_{n}$ is a normalization constant. ${ }^{11}$

- $\mu_{n}$ is the 'renormalized' chemical potential. ${ }^{12}$ It is close to $L^{2 n} \mu_{0}$.

- For each pair in the polydisc

$$
\left\{\left(\psi_{*}, \psi\right) \in \mathbb{C}^{\mathcal{X}_{0}^{(n)}} \times \mathbb{C}^{\mathcal{X}_{0}^{(n)}}|| \psi_{(*)}(x) \mid \leq \kappa_{n} \text { for all } x \in \mathcal{X}_{0}^{(n)}\right\}
$$

the fields $\phi_{* n}\left(\psi_{*}, \psi\right)(u), \phi_{n}\left(\psi_{*}, \psi\right)(u)$ on $\mathcal{X}_{n}$ are critical points of the functional

$$
\begin{aligned}
\left(\phi_{*}, \phi\right) & \mapsto A_{n}\left(\psi_{*}, \psi, \phi_{*}, \phi\right) \\
= & \left.a_{n}\left\langle\left(\psi_{*}-Q_{n} \phi_{*}\right),\left(\psi-Q_{n} \phi\right)\right\rangle_{0}-\left\langle\phi_{*},\left(\partial_{0}+\Delta+\mu_{n}\right) \phi\right)\right\rangle_{n} \\
& +\mathcal{V}_{n}\left(\phi_{*}, \phi\right) .
\end{aligned}
$$

The maps $\phi_{* n}\left(\psi_{*}, \psi\right), \phi_{n}\left(\psi_{*}, \psi\right)$ are holomorphic on that polydisc.

In practical terms, what have we achieved? If $\psi=z$ is a constant field on $\mathcal{X}_{0}$, then the dominant part of the initial effective potential is

$$
\begin{aligned}
A_{0}\left(\psi^{*}, \psi\right) & =\mathcal{V}_{0}\left(\psi^{*}, \psi\right)-\mu_{0}\left\langle\psi^{*}, \psi\right\rangle_{0}=\left|\mathcal{X}_{0}\right|\left(\frac{\mathrm{v}_{0}}{2}|z|^{4}-\mu_{0}|z|^{2}\right) \\
& =\left|\mathcal{X}_{0}\right| \frac{\mathrm{v}_{0}}{2}\left[\left(|z|^{2}-\frac{\mu_{0}}{\mathrm{v}_{0}}\right)^{2}-\frac{\mu_{0}^{2}}{\mathrm{v}_{0}^{2}}\right]
\end{aligned}
$$

where, $\mathrm{v}_{0}=\sum_{\mathcal{X}_{0}^{3}} V_{0}\left(0, x_{2}, x_{3}, x_{4}\right)$. The graph of the real-valued function

$$
\frac{\mathrm{v}_{0}}{2}\left[\left(|z|^{2}-\frac{\mu_{0}}{\mathrm{v}_{0}}\right)^{2}-\frac{\mu_{0}^{2}}{\mathrm{v}_{0}^{2}}\right]
$$

over the complex plane $z=x_{1}+\imath x_{2}$ is a surface of revolution around the $x_{3}$-axis with the circular well of absolute minima $|z|=\sqrt{\frac{\mu_{0}}{\mathrm{v}_{0}}}$. Our hypothesis on $\mu_{0}$ implies that the radius and depth of the well are of order one and order $\mathrm{v}_{0}$, respectively. After $n$ renormalization group steps, the effective potential becomes

$$
\begin{aligned}
& \left.A_{n}\left(\psi^{*}, \psi, \phi_{* n}\left(\psi^{*}, \psi\right), \phi_{n}\left(\psi^{*}, \psi\right)\right)\right|_{\psi=z} \\
& \quad \approx\left|\mathcal{X}_{0}^{(n)}\right| \frac{\mathrm{v}_{0}}{2 L^{n}}\left[\left(|z|^{2}-\frac{\mu_{n}}{\mathrm{v}_{0} / L^{n}}\right)^{2}-\frac{\mu_{n}^{2}}{\left(\mathrm{v}_{0} / L^{n}\right)^{2}}\right]
\end{aligned}
$$

\footnotetext{
${ }^{10}$ It is necessary to measure the size of $p_{n}$ by introducing an appropriate norm. See the last paragraphs of this overview.

${ }^{11}$ When we take logarithms and ultimately differentiate with respect to an external field to obtain correlation functions, it will disappear.

${ }^{12}$ We will describe the inductive construction of $\mu_{n}$ later on in this overview. The dependence of $p_{n}$ on the derivatives of the fields arises because of the renormalization of the chemical potential.
} 
since, by [16, Remark 1.1], $\left.\phi_{n}\left(\psi^{*}, \psi\right)\right|_{\psi=z} \approx z$ and $\left.\phi_{* n}\left(\psi^{*}, \psi\right)\right|_{\psi=z} \approx z^{*}$. The graph is again a surface of revolution with the circular well of absolute minima $|z|=\sqrt{\frac{\mu_{n}}{\mathrm{v}_{0} / L^{n}}}$, but now the radius and depth are of order $L^{\frac{3}{2} n}$ and order $L^{5 n} \mathrm{v}_{0}$, respectively; the well is developing. We stop the flow when the well becomes so wide and so deep that we can no longer construct background fields by expanding around $\psi_{*}, \psi=0$. This happens as $\mu_{n}$ approaches order one.

If the power series expansion of the perturbative correction $p_{n}$ had a quadratic part $\sum_{x, y \in \mathcal{X}_{0}^{(n)}} K(x, y) \psi_{*}(x) \psi(y)$ the discussion of the evolving well in the last paragraph would be misleading, because the minimum of the total action $A_{n}-p_{n}$ would not be close enough to the minimum of the dominant part $A_{n}$. The requirement that $p_{n}$ must not contain quadratic terms is the renormalization condition for the chemical potential. (See, Step 9 below.) Under the scaling map (3), the local monomials

$$
\left\langle\psi_{*}, \psi\right\rangle_{0} \quad\left\langle\psi_{*}, \partial_{\nu} \psi\right\rangle_{0} \quad 1 \leq \nu \leq 3
$$

are relevant, and the local monomials

$$
\left\langle\psi_{*}, \partial_{0} \psi\right\rangle_{0} \quad\left\langle\partial_{\nu} \psi_{*}, \partial_{\nu^{\prime}} \psi\right\rangle_{0} 1 \leq \nu, \nu^{\prime} \leq 3
$$

are marginal. The local monomials $\left\langle\psi_{*}, \partial_{\nu} \psi\right\rangle_{0}, 1 \leq \nu \leq 3$, do not appear, because of reflection invariance. See [13, Definition B.1 and Lemma B.4]. So $p_{n}$ does not contain any relevant monomials.

The parabolic renormalization group flow drives the system away from the trivial (noninteracting) fixed point. To continue, we will have to construct background fields by expanding about configurations supported near the bottom of the developing well, analogously to the 'Bogoliubov Ansatz.' At present, we expect to continue the parabolic flow, but expanding about configurations supported near the bottom of the well, through a transition regime (which overlaps with the regime of $[13,14]$ ) until $\mu_{n}$ becomes large enough (but still of order one), and then switch to a new 'elliptic' renormalization group flow for the push to the symmetry broken, superfluid fixed point. In Appendix A, below, we perform several model computations that contrast the parabolic nature of the early renormalization group steps with the elliptical nature of the late renormalization group steps.

The next part of this overview is an outline, in nine steps, of the inductive construction that uses a steepest descent/stationary phase calculation to build the desired form for $F_{n+1}\left(\psi_{*}, \psi\right)=B_{n+1}\left(\mathbb{S}^{-1} \psi_{*}, \mathbb{S}^{-1} \psi\right)$, from that of $F_{n}\left(\psi_{*}, \psi\right), n \geq 0$, where

$$
B_{n+1}\left(\theta_{*}, \theta\right)=\frac{1}{N^{(n)}} \int_{\mathbb{C}^{\mathcal{X}_{0}^{(n)}}} \prod_{x \in \mathcal{X}_{0}^{(n)}} \frac{d \psi(x)^{*} \wedge d \psi(x)}{2 \pi \imath} e^{-\frac{1}{L^{2}}\left\langle\theta_{*}-Q \psi^{*}, \theta-Q \psi\right\rangle}{ }_{-1} F_{n}\left(\psi^{*}, \psi\right) .
$$


We are expecting that, by induction,

$$
\begin{aligned}
B_{n+1}\left(\theta_{*}, \theta\right) & \frac{1}{N^{(n)}} \int_{S_{n}} \prod_{x \in \mathcal{X}_{0}^{(n)}} \frac{d \psi(x)^{*} \wedge d \psi(x)}{2 \pi \imath} e^{-\frac{1}{L^{2}}\left\langle\theta_{*}-Q \psi^{*}, \theta-Q \psi\right\rangle_{-1}} F_{n}^{(S F)}\left(\psi^{*}, \psi\right) \\
& +O\left(e^{-1 / \mathfrak{v}_{0}^{\varepsilon}}\right) \\
= & \frac{1}{N^{(n)} \mathcal{Z}_{n}} \int_{S_{n}} \prod_{x \in \mathcal{X}_{0}^{(n)}} \frac{d \psi(x)^{*} \wedge d \psi(x)}{2 \pi \imath} e^{-\frac{1}{L^{2}}\left\langle\theta_{*}-Q \psi^{*}, \theta-Q \psi\right\rangle_{-1}-A_{n}\left(\psi^{*}, \psi, \phi_{* n}, \phi_{n}\right)+p_{n}} \\
& +O\left(e^{-1 / \mathfrak{v}_{0}^{\varepsilon}}\right) \\
= & \text { Dominant Part }+ \text { Non Perturbative Correction. }
\end{aligned}
$$

We emphasize that Steps 1 and 6, which control the difference between $F_{n+1}\left(\psi_{*}, \psi\right)$ and its, dominant, 'small field,' part $F_{n+1}^{(S F)}\left(\psi_{*}, \psi\right)$, have not been proven, though we do supply some motivation in [15].

Step 1 (Large field generates small factors). If $\Psi \in \mathbb{C}^{\mathcal{X}_{0}^{(n+1)}}$ is 'large field,' that is $\Psi \notin S_{n+1}$, then we expect $B_{n+1}\left(\mathbb{S}^{-1} \Psi^{*}, \mathbb{S}^{-1} \Psi\right)=O\left(e^{-1 / \mathfrak{v}_{0}^{\varepsilon}}\right)$, since the real part of the exponent appearing in the integrand of $(6)$ is of order $-\frac{1}{\mathfrak{v}_{0}^{\varepsilon}}$. See [15, Proposition 1, 'Corollary' 2 and the subsequent Steps 1 and 2].

We assume for the rest of the discussion that $\Psi \in \mathbb{C}^{\mathcal{X}_{0}^{(n+1)}}$ is 'small field,' that is $\Psi \in S_{n+1}$, and therefore construct holomorphic functions of $\left(\Psi_{*}, \Psi\right)$ on the product $S_{n+1} \times S_{n+1}$. Let $\theta_{*}=\mathbb{S}^{-1} \Psi_{*}$ and $\theta=\mathbb{S}^{-1} \Psi$.

Step 2 (Holomorphic form representation). We wish to analyze the integral in (6) by a steepest descent/stationary phase argument. Recall that a critical point of a function $f(z)$ of one complex variable $z=x+i y$, that is not analytic in $z$, is a point where both partial derivatives $\frac{\partial f}{\partial x}$ and $\frac{\partial f}{\partial y}$, or equivalently, both partial derivatives $\frac{\partial f}{\partial z}=\frac{1}{2}\left(\frac{\partial}{\partial x}-i \frac{\partial}{\partial y}\right) f$ and $\frac{\partial f}{\partial \bar{z}}=\frac{1}{2}\left(\frac{\partial}{\partial x}+i \frac{\partial}{\partial y}\right) f$ vanish. We prefer the latter formulation. So we rewrite the integral in (6) in a form that allows us to treat $\psi$ and its complex conjugate as independent fields. For each fixed $\left(\theta_{*}, \theta\right)$, the 'action'

$$
\begin{aligned}
\mathcal{A}_{n}\left(\theta_{*}, \theta, \psi_{*}, \psi\right) & \\
= & \left\{-\frac{1}{L^{2}}\left\langle\theta_{*}-Q \psi_{*}, \theta-Q \psi\right\rangle_{-1}-A_{n}\left(\psi_{*}, \psi, \phi_{* n}\left(\psi_{*}, \psi\right), \phi_{n}\left(\psi_{*}, \psi\right)\right)\right\} \\
& \quad+p_{n}\left(\psi_{*}, \psi, \nabla \psi_{*}, \nabla \psi\right) \\
= & -A_{n, \mathrm{eff}}\left(\theta_{*}, \theta, \psi_{*}, \psi\right)+p_{n}\left(\psi_{*}, \psi, \nabla \psi_{*}, \nabla \psi\right)
\end{aligned}
$$

is a holomorphic function of $\left(\psi_{*}, \psi\right)$ on $S_{n} \times S_{n}$. By design, the Dominant Part of $B_{n+1}\left(\theta_{*}, \theta\right)$ in (6) is expressed as (a constant times) the integral of the holomorphic form 


$$
e^{\mathcal{A}_{n}\left(\theta_{*}, \theta, \psi_{*}, \psi\right)} \bigwedge_{x \in \mathcal{X}_{0}^{(n)}} \frac{d \psi_{*}(x) \wedge d \psi(x)}{2 \pi \imath}
$$

of degree $2\left|\mathcal{X}_{0}^{(n)}\right|$ over the real subspace in $S_{n} \times S_{n}$ given by $\psi_{*}=\psi^{*}$. We shall see below that, typically, the critical point does not lie in the real subspace and so is not in the domain of integration. This representation permits us to use Stokes' theorem, ${ }^{13}$ to shift the contour of integration to a non-real contour that does contain the critical point of (the principal terms of) the action. The shift will be implemented in Step 6 .

Step 3 (Critical Points). Our next task is to find critical points. In (7), above, we wrote the exponent, $\mathcal{A}_{n}\left(\theta_{*}, \theta, \psi_{*}, \psi\right)$, as the sum of a very explicit, main, part - $A_{n, \text { eff }}$ and a not very explicit, smaller, part $p_{n}$. We just find the critical points of $A_{n \text {,eff }}$ rather than the full $\mathcal{A}_{n}$. Indeed, there is a unique pair of holomorphic maps ${ }^{14} \psi_{* \mathrm{cr}}\left(\theta_{*}, \theta\right), \psi_{\mathrm{cr}}\left(\theta_{*}, \theta\right)$ from $\left(\mathbb{S}^{-1} S_{n+1}\right) \times\left(\mathbb{S}^{-1} S_{n+1}\right)$ to $S_{n}$ such that the gradient $\left(\begin{array}{l}\nabla_{\psi_{*}} \\ \nabla_{\psi}\end{array}\right)$ of $A_{n \text {,eff }}\left(\theta_{*}, \theta, \psi_{*}, \psi\right)$ vanishes when $\psi_{*}=\psi_{* \mathrm{cr}}\left(\theta_{*}, \theta\right), \psi=\psi_{\mathrm{cr}}\left(\theta_{*}, \theta\right)$. This pair of 'critical field maps' can be constructed by solving the critical point equations, a nonlinear parabolic system of (discrete) partial difference equations, using the natural contraction mapping argument to perturb off of the linearized equations. ${ }^{15}$ The analysis of the linearized equations is based on a careful examination of some linear operators given in [10]. Beware that, in general, $\psi_{* \mathrm{cr}}\left(\theta^{*}, \theta\right) \neq \psi_{\mathrm{cr}}\left(\theta^{*}, \theta\right)^{*}$.

To start the stationary phase calculation, we factor the integral of the holomorphic form (8) over the real subspace $\left\{\left(\psi_{*}, \psi\right) \in S_{n} \times S_{n} \mid \psi_{*}=\psi^{*}\right\}$ as the product of

$$
e^{\mathcal{A}_{n}\left(\theta_{*}, \theta, \psi_{* \mathrm{cr}}\left(\theta_{*}, \theta\right), \psi_{\mathrm{cr}}\left(\theta_{*}, \theta\right)\right)}
$$

and the 'fluctuation integral'

$$
\int_{\substack{\text { real } \\ S_{n} \times S_{n}}} e^{\mathcal{A}_{n}\left(\theta_{*}, \theta, \psi_{*}, \psi\right)-\mathcal{A}_{n}\left(\theta_{*}, \theta, \psi_{* \mathrm{cr}}\left(\theta_{*}, \theta\right), \psi_{\mathrm{cr}}\left(\theta_{*}, \theta\right)\right)} \bigwedge_{x \in \mathcal{X}_{0}^{(n)}} \frac{d \psi_{*}(x) \wedge d \psi(x)}{2 \pi \imath} .
$$

Step 4 (The Value of the Action at the Critical Point). We would expect that the biggest contribution to the integral would come from simply evaluating the exponent at the critical point, and that the biggest contribution to the value of the exponent $\mathcal{A}_{n}$ at the critical point would come from evaluating $-A_{n \text {,eff }}$

\footnotetext{
13 The argument is similar to the use of Cauchy's theorem in stationary phase arguments for functions of one variable.

14 In $[13,14]$ these maps are called $\psi_{n *}, \psi_{n}$.

15 In $[13,14,16]$ we take another route to the critical field maps. The background fields $\phi_{(*) n}\left(\psi_{*}, \psi\right)$ are constructed first, using the natural contraction mapping argument to perturb off of the linearized background field equations. See [16, Proposition 2.1]. The critical fields can then be expressed as functions of the background fields. See [13, Proposition 3.4].
} 
at the critical point. By [13, Proposition 3.4.c]

$$
\begin{array}{r}
\left.A_{n, \mathrm{eff}}\left(\theta_{*}, \theta, \psi_{*}, \psi\right)\right|_{\psi_{*}=\psi_{* \mathrm{cr}}\left(\theta_{*}, \theta\right), \psi=\psi_{\mathrm{cr}}\left(\theta_{*}, \theta\right)} \\
=\check{A}_{n+1}\left(\theta_{*}, \theta, \check{\phi}_{* n+1}\left(\theta_{*}, \theta\right), \check{\phi}_{n+1}\left(\theta_{*}, \theta\right)\right)
\end{array}
$$

where

$$
\begin{aligned}
\check{A}_{n+1}\left(\theta_{*}, \theta, f_{*}, f\right)= & \frac{a_{n+1}}{L^{2}}\left\langle\theta_{*}-Q Q_{n} f_{*}, \theta-Q Q_{n} f\right\rangle_{-1} \\
& -\left\langle f_{*},\left(\partial_{0}+\Delta+\mu_{n}\right) f\right\rangle_{n}+\mathcal{V}_{n}\left(f_{*}, f\right)
\end{aligned}
$$

and the 'checked' field

$$
\check{\phi}_{(*) n+1}\left(\theta_{*}, \theta\right)=\phi_{(*) n}\left(\psi_{* \mathrm{cr}}\left(\theta_{*}, \theta, \psi_{\mathrm{cr}}\left(\theta_{*}, \theta\right)\right)\right.
$$

is the background field evaluated at the critical point. Consequently,

$$
\begin{aligned}
& e^{\mathcal{A}_{n}\left(\theta_{*}, \theta, \psi_{* \mathrm{cr}}\left(\theta_{*}, \theta\right), \psi_{\mathrm{cr}}\left(\theta_{*}, \theta\right)\right)} \\
& \quad=e^{-\check{A}_{n+1}\left(\theta_{*}, \theta, \check{\phi}_{* n+1}\left(\theta_{*}, \theta\right), \check{\phi}_{n+1}\left(\theta_{*}, \theta\right)\right)+p_{n}\left(\psi_{* \mathrm{cr}}, \psi_{\mathrm{cr}}, \nabla \psi_{* \mathrm{cr}}, \nabla \psi_{\mathrm{cr}}\right)} .
\end{aligned}
$$

Remark. Bear in mind that the checked fields depend implicitly on $\mu_{n}$. In the next steps, we will build a new 'renormalized' chemical potential $\mu_{n+1}$ that will appear in $A_{n+1}$. If, for the purposes of discussion, we ignored the effects of renormalization, $A_{n+1}$ would just be a rescaled $\check{A}_{n+1}$ (see [13, Definition 2.3 and Lemma 2.4.c]) and the new background field $\phi_{(*) n+1}$ would just be a rescaled $\check{\phi}_{(*) n+1}$ (see [13, Definition 3.2 and Proposition 3.4.b]). So, we are not far off.

Step 5 (Diagonalization of the Quadratic Form in the Fluctuation Integral). Next consider the fluctuation integral (9). Make the change variables

$$
\left(\psi_{*}, \psi\right) \rightarrow\left(\delta \psi_{*}=\psi_{*}-\psi_{* \mathrm{cr}}\left(\theta_{*}, \theta\right), \delta \psi=\psi-\psi_{\mathrm{cr}}\left(\theta_{*}, \theta\right)\right)
$$

to shift the critical point to $\delta \psi_{*}=\delta \psi=0$. Substitute $\psi_{(*)}=\psi_{(*) \mathrm{cr}}\left(\theta_{*}, \theta\right)+$ $\delta \psi_{(*)}$ into the main part

$$
A_{n, \mathrm{eff}}\left(\theta_{*}, \theta, \psi_{*}, \psi\right)-A_{n, \mathrm{eff}}\left(\theta_{*}, \theta, \psi_{* \mathrm{cr}}\left(\theta_{*}, \theta\right), \psi_{\mathrm{cr}}\left(\theta_{*}, \theta\right)\right)
$$

of the exponent and expand in powers of $\delta \psi_{(*)}$. The constant and, by criticality, linear parts vanish. The quadratic term has a dominant part (see [13, Lemma $4.1,(4.13)]$ and $\left[14\right.$, Lemma 5.5]), that is independent of $\theta_{*}, \theta$. All of the eigenvalues of the kernel of that dominant part are bounded away from the negative real axis, uniformly in $n .{ }^{16}$ So, it is invertible and its inverse, $C^{(n)}$, has a square root $D^{(n)}$, all of whose eigenvalues have strictly positive real parts. See [10, Corollary 4.5]. Now, the Taylor expansion of the above difference of effective actions in the new variables $\delta \psi_{*}=D^{(n)^{T}} \zeta_{*}, \delta \psi=D^{(n)} \zeta$ becomes

$$
\begin{aligned}
& \left\langle\zeta_{*}, \zeta\right\rangle_{0}+\text { smaller terms of degree } 2 \text { in } \zeta_{*}, \zeta \\
& + \text { terms of degree at least } 3 \mathrm{in} \zeta_{*}, \zeta
\end{aligned}
$$

16 A major part of [10] is devoted to proving this vital technical statement. 
and the fluctuation integral (9) becomes

$$
\int_{\Omega_{n}\left(\theta_{*}, \theta\right)} e^{-\left\langle\zeta_{*}, \zeta\right\rangle_{0}+q_{n}\left(\theta_{*}, \theta, \zeta_{*}, \zeta\right)} \operatorname{det}\left(D^{(n)}\right)^{2} \bigwedge_{x \in \mathcal{X}_{0}^{(n)}} \frac{d \zeta_{*}(x) \wedge d \zeta(x)}{2 \pi \imath}
$$

where the domain of integration $\Omega_{n}\left(\theta_{*}, \theta\right)$ consists of the set of all pairs $\left(\zeta_{*}, \zeta\right) \in \mathbb{C}^{\mathcal{X}_{0}^{(n)}} \times \mathbb{C}^{\mathcal{X}_{0}^{(n)}}$ such that $\left(\psi_{* \operatorname{cr}}\left(\theta_{*}, \theta\right)+D^{(n)^{T}} \zeta_{*}, \psi_{\mathrm{cr}}\left(\theta_{*}, \theta\right)+D^{(n)} \zeta\right)$ is in the real subspace of $S_{n} \times S_{n}$. The term $q_{n}$ is holomorphic on the complex domain of all quadruples $\left(\theta_{*}, \theta, \zeta_{*}, \zeta\right)$ with $\left(\theta_{*}, \theta\right) \in\left(\mathbb{S}^{-1} S_{n+1}\right) \times\left(\mathbb{S}^{-1} S_{n+1}\right)$ and

$$
\left(\psi_{* \mathrm{cr}}\left(\theta_{*}, \theta\right)+D^{(n)^{T}} \zeta_{*}, \psi_{\mathrm{cr}}\left(\theta_{*}, \theta\right)+D^{(n)} \zeta\right) \in S_{n} \times S_{n}
$$

See [13, (4.4) and Corollary 4.3].

Step 6 (Stokes' Theorem). For each pair $\left(\theta_{*}, \theta\right) \in\left(\mathbb{S}^{-1} S_{n+1}\right) \times\left(\mathbb{S}^{-1} S_{n+1}\right)$, we construct, in $[15$, following $(22)]$, a $2\left|\mathcal{X}_{0}^{(n)}\right|+1$ (real) dimensional 'cylinder,' inside the $\left(\zeta_{*}, \zeta\right)$ domain of analyticity of $q_{n}$, whose boundary consists of

○ the original domain of integration $\Omega_{n}\left(\theta_{*}, \theta\right)$ (which typically does not contain the critical point $\zeta=\zeta_{*}=0$ ),

$\circ$ the desired new domain of integration

$$
D_{n}=\left\{\left(\zeta_{*}, \zeta\right)\left|\zeta_{*}=\zeta^{*},\right| \zeta(x) \mid<\frac{1}{4}\left(\frac{L^{n+1}}{\mathfrak{v}_{0}}\right)^{\varepsilon / 2} \text { for all } x \in \mathcal{X}_{0}^{(n)}\right\}
$$

(which does contain the critical point $\zeta=\zeta_{*}=0$ )

$\circ$ and components on which $e^{-\left\langle\zeta_{*}, \zeta\right\rangle_{0}+q_{n}\left(\theta_{*}, \theta, \zeta_{*}, \zeta\right)}$ is $O\left(e^{-1 / \mathfrak{v}_{0}^{\varepsilon}}\right)$.

See $[15,(23)]$. The holomorphic differential form in Step 5 has maximal rank and is therefore closed. It follows from Stokes' theorem that the fluctuation integral (10) is equal to the small field contribution

$$
\check{\mathcal{F}}_{n}\left(\theta_{*}, \theta\right)=\operatorname{det}\left(D^{(n)}\right)^{2} \int_{D_{n}} \prod_{x \in \mathcal{X}_{0}^{(n)}} \frac{d \zeta(x)^{*} \wedge d \zeta(x)}{2 \pi \imath} e^{-\left\langle\zeta^{*}, \zeta\right\rangle_{0}+q_{n}\left(\theta_{*}, \theta, \zeta^{*}, \zeta\right)}
$$

plus corrections that are expected to be nonperturbatively small.

Step 7 (The Logarithm of the Fluctuation Integral). In [5], we developed a simple variant of the polymer expansion that can be directly applied to the integral in (11) to obtain the logarithm $\log \left[\frac{\check{\mathcal{F}}_{n}\left(\theta_{*}, \theta\right)}{\check{\mathcal{F}}_{n}(0,0)}\right]$ as an analytic function on $\left(\mathbb{S}^{-1} S_{n+1}\right) \times\left(\mathbb{S}^{-1} S_{n+1}\right)$. See [14, Proposition 5.6].

Step 8 (Rescaling). To this point, we have determined that the small field part of $B_{n+1}\left(\theta_{*}, \theta\right)$ is a constant times the exponential of the sum of

○ the contribution which comes from simply evaluating $\mathcal{A}_{n}$ at the critical point - in Step 4, we saw that this was

$$
-\check{A}_{n+1}\left(\theta_{*}, \theta, \check{\phi}_{* n+1}\left(\theta_{*}, \theta\right), \check{\phi}_{n+1}\left(\theta_{*}, \theta\right)\right)+p_{n}\left(\psi_{* \mathrm{cr}}, \psi_{\mathrm{cr}}, \nabla \psi_{* \mathrm{cr}}, \nabla \psi_{\mathrm{cr}}\right)
$$

$\circ$ and an analytic function that came, in Step 7, from the fluctuation integral. 
We are now ready to scale to get the small field part of

$$
F_{n+1}\left(\Psi_{*}, \Psi\right)=B_{n+1}\left(\mathbb{S}^{-1} \Psi_{*}, \mathbb{S}^{-1} \Psi\right)
$$

Using that

$$
\begin{aligned}
\frac{1}{L^{2}}\left\langle\mathbb{S}^{-1} \Psi_{*}, \mathbb{S}^{-1} \Psi\right\rangle_{-1} & =\left\langle\Psi_{*}, \Psi\right\rangle_{0} \\
\mathbb{S} Q Q_{n} \mathbb{S}^{-1} & =Q_{n+1} \\
\left\langle\mathbb{S}^{-1} f_{*}, \mathbb{S}^{-1} f\right\rangle_{n} & =L^{2}\left\langle f_{*}, f\right\rangle_{n+1} \\
\left\langle\mathbb{S}^{-1} f_{*},\left(\partial_{0}+\Delta\right) \mathbb{S}^{-1} f\right\rangle_{n} & =\left\langle f,\left(\partial_{0}+\Delta\right) f\right\rangle_{n+1}
\end{aligned}
$$

(see [13, Remark 2.2.c and Lemma 2.4.a,b]) we have that

$$
\begin{gathered}
\left.\check{A}_{n+1}\left(\theta_{*}, \theta, \check{\phi}_{* n+1}\left(\theta_{*}, \theta\right), \check{\phi}_{n+1}\left(\theta_{*}, \theta\right)\right)\right|_{\theta_{(*)}=\mathbb{S}^{-1} \Psi_{(*)}} \\
=A_{n+1}^{\prime}\left(\Psi_{*}, \Psi, \phi_{* n+1}^{\prime}\left(\Psi_{*}, \Psi\right), \phi_{n+1}^{\prime}\left(\Psi_{*}, \Psi\right)\right)
\end{gathered}
$$

where

$$
\begin{aligned}
A_{n+1}^{\prime}\left(\Psi_{*}, \Psi, f_{*}, f\right)= & \check{A}_{n+1}\left(\mathbb{S}^{-1} \Psi_{*}, \mathbb{S}^{-1} \Psi, \mathbb{S}^{-1} f_{*}, \mathbb{S}^{-1} f\right) \\
= & a_{n+1}\left\langle\Psi_{*}-Q_{n+1} f_{*}, \Psi-Q_{n+1} f\right\rangle_{0} \\
& -\left\langle f_{*},\left(\partial_{0}+\Delta+L^{2} \mu_{n}\right) f\right\rangle_{n+1}+\mathcal{V}_{n+1}^{\prime}\left(f_{*}, f\right) \\
\phi_{(*) n+1}^{\prime}\left(\Psi_{*}, \Psi\right)= & \mathbb{S} \check{\phi}_{(*) n+1}\left(\mathbb{S}^{-1} \Psi_{*}, \mathbb{S}^{-1} \Psi\right)
\end{aligned}
$$

and if the kernel $V_{n}$ of $\mathcal{V}_{n}$ were exactly the $V_{n}^{(u)}$ of (4), then the kernel of $\mathcal{V}_{n+1}^{\prime}$ would be exactly $V_{n+1}^{(u)}$. See [13, Remark 2.2.h]. Renormalization is going to tweak, for example, the value of the chemical potential. As a result, $A_{n+1}^{\prime}$ is not quite $A_{n+1}$, and $\phi_{(*) n+1}^{\prime}$ is not quite $\phi_{(*) n+1}$. That's the reason for putting the primes on.

Similarly, the contributions from $p_{n}\left(\psi_{* \mathrm{cr}}, \psi_{\mathrm{cr}}, \nabla \psi_{* \mathrm{cr}}, \nabla \psi_{\mathrm{cr}}\right)$ and from the fluctuation integral get scaled to

$$
\begin{aligned}
p_{n+1}^{\prime} & \left(\Psi_{*}, \Psi,\left\{\Psi_{* \nu}\right\}_{\nu=0}^{3},\left\{\Psi_{\nu}\right\}_{\nu=0}^{3}\right) \\
= & {\left[p_{n}\left(\psi_{* \mathrm{cr}}\left(\theta_{*}, \theta\right), \psi_{\mathrm{cr}}\left(\theta_{*}, \theta\right), \nabla \psi_{* \mathrm{cr}}\left(\theta_{*}, \theta\right), \nabla \psi_{\mathrm{cr}\left(\theta_{*}, \theta\right)}\right)\right.} \\
& \left.+\log \left(\frac{\check{\mathcal{F}}_{n}\left(\mathbb{S}^{-1} \Psi_{*}, \mathbb{S}^{-1} \Psi\right)}{\check{\mathcal{F}}_{n}(0,0)}\right)\right]_{\theta_{(*)}=\mathbb{S}^{-1} \Psi_{(*)}}
\end{aligned}
$$

and we have that, renaming $\Psi_{(*)}$ to $\psi_{(*)}$, the small field part of $F_{n+1}\left(\psi_{*}, \psi\right)$ is

$$
F_{n+1}^{(S F)}\left(\psi_{*}, \psi\right)=e^{-A_{n+1}^{\prime}\left(\psi_{*}, \psi, \phi_{* n+1}^{\prime}\left(\psi_{*}, \psi\right), \phi_{n+1}^{\prime}\left(\psi_{*}, \psi\right)\right)+p_{n+1}^{\prime}\left(\psi_{*}, \psi, \nabla \psi^{*}, \nabla \psi\right)}
$$

on $S_{n+1} \times S_{n+1}$.

Step 9 (Renormalization of the Chemical Potential). At this point, we are close to the end of the induction step, but not there yet because the power series $p_{n+1}^{\prime}$ contains (renormalization group) relevant contributions, in particular a quadratic term $\left\langle\psi_{*}, K \psi\right\rangle_{0}$, where $K$ is a translation and (spatial) reflection invariant linear operator mapping $\mathbb{C}^{\mathcal{X}_{0}^{(n+1)}}$ to itself. If such a term were to be 
left in $p_{n+1}$ it would, by the third line of (12), grow by roughly a factor of $L^{2}$ in each future renormalization group step. So we need to move (at least the local part of) this term out of $p_{n+1}$ and into $A_{n+1}$. By the discrete fundamental theorem of calculus, for any translation invariant $K$,

$$
\left\langle\psi_{*}, K \psi\right\rangle_{0}=\mathcal{K}\left\langle\psi_{*}, \psi\right\rangle_{0}+\sum_{\nu=0}^{3}\left\langle\psi_{*}, K^{\nu}\left(\partial_{\nu} \psi\right)\right\rangle_{0}
$$

where $\mathcal{K} \in \mathbb{C}$ and $K^{\nu}, \nu=0,1,2,3$, are linear operators on $\mathbb{C}^{\mathcal{X}_{0}^{(n+1)}}$. See $[14$, Corollary B.2]. By reflection invariance, $\mathcal{K}$ is real and $\sum_{\nu=1}^{3}\left\langle\psi_{*}, K^{\nu}\left(\partial_{\nu} \psi\right)\right\rangle_{0}$ can be rewritten as a sum of marginal and irrelevant monomials. See [14, Lemma B.3.c].

So we would like to move $\mathcal{K}\left\langle\psi_{*}, \psi\right\rangle_{0}$ out of $p_{n+1}$ into $A_{n+1}$. There are two factors that complicate (but not seriously) this move.

○ The chemical potential term in $A_{n+1}^{\prime}\left(\psi_{*}, \psi, \phi_{* n+1}^{\prime}\left(\psi_{*}, \psi\right), \phi_{n+1}^{\prime}\left(\psi_{*}, \psi\right)\right)$ is

$$
L^{2} \mu_{n}\left\langle\phi_{* n+1}^{\prime}\left(\psi_{*}, \psi\right), \phi_{n+1}^{\prime}\left(\psi_{*}, \psi\right)\right\rangle_{n+1} .
$$

It is expressed in terms of $\phi_{(*) n+1}^{\prime}\left(\psi_{*}, \psi\right)$ rather than directly in terms of $\psi_{(*)}$.

○ The prime fields $\phi_{* n+1}^{\prime}\left(\psi_{*}, \psi\right), \phi_{n+1}^{\prime}\left(\psi_{*}, \psi\right)$ are background fields with chemical potential $L^{2} \mu_{n}$, not with the chemical potential $\mu_{n+1}$ that we are going to end up with (and which we do not yet know).

To deal with the first complication, we use that $\phi_{(*) n+1}^{\prime}\left(\psi_{*}, \psi\right)=\mathrm{B}_{(*)} \psi_{(*)}$ plus terms of degree at least three in $\left(\psi_{*}, \psi\right)$ (see [16, Proposition 2.1.a]). Because the linear operators $\mathrm{B}_{(*)}$ have left inverses (see [10, Lemma 5.7] and the beginning of the proof of $[14$, Lemma 6.3$]$ ), one can show that ${ }^{17} \mathcal{K}\left\langle\psi_{*}, \psi\right\rangle_{0}=$ $\mathcal{K}^{\prime}\left\langle\phi_{* n+1}^{\prime}\left(\psi_{*}, \psi\right), \phi_{n+1}^{\prime}\left(\psi_{*}, \psi\right)\right\rangle_{n+1}$ plus a power series in $\psi_{*}, \psi, \nabla \psi_{*}, \nabla \psi$ that converges on the desired domain of analyticity and that does not contain any relevant contributions. See [14, Lemma 6.3]. Thus,

$$
p_{n+1}^{\prime}=\mathcal{K}^{\prime}\left\langle\phi_{* n+1}^{\prime}, \phi_{n+1}^{\prime}\right\rangle_{n+1}+p_{n+1}^{\prime \prime}
$$

where $p_{n+1}^{\prime \prime}$ has no $\left\langle\psi_{*}, \psi\right\rangle_{0}$ term. Moving $\mathcal{K}^{\prime}\left\langle\phi_{* n+1}^{\prime}, \phi_{n+1}^{\prime}\right\rangle_{n+1}$ from $p_{n+1}^{\prime}$ into $A_{n+1}^{\prime}$, we obtain

$$
-A_{n+1}^{\prime}\left(\psi^{*}, \psi, \phi_{* n+1}^{\prime}, \phi_{n+1}^{\prime}\right)+p_{n+1}^{\prime}=-A_{n+1}^{\prime \prime}\left(\psi^{*}, \psi, \phi_{* n+1}^{\prime}, \phi_{n+1}^{\prime}\right)+p_{n+1}^{\prime \prime}
$$

with

$$
\begin{aligned}
A_{n+1}^{\prime \prime}\left(\psi^{*}, \psi, f_{*}, f\right)= & a_{n+1}\left\langle\psi_{*}-Q_{n+1} f_{*}, \psi-Q_{n+1} f\right\rangle_{0} \\
& -\left\langle f_{*},\left(\partial_{0}+\Delta+\left(L^{2} \mu_{n}+\mathcal{K}^{\prime}\right) f\right\rangle_{n+1}+\mathcal{V}_{n+1}^{\prime}\left(f_{*}, f\right) .\right.
\end{aligned}
$$

But we are still not done - we still have the second complication to deal with. The prime fields $\phi_{* n+1}^{\prime}\left(\psi_{*}, \psi\right), \phi_{n+1}^{\prime}\left(\psi_{*}, \psi\right)$ are background fields for

17 For reasons that will be explained shortly, we do not actually use this fact expressed in this way. 
chemical potential $L^{2} \mu_{n}$, and not for chemical potential $L^{2} \mu_{n}+\mathcal{K}^{\prime}$. That is, the prime fields are critical for $f_{*}, f \mapsto A_{n+1}^{\prime}\left(\psi_{*}, \psi, f_{*}, f\right)$ and not for $f_{*}, f \mapsto A_{n+1}^{\prime \prime}\left(\psi_{*}, \psi, f_{*}, f\right)$, as they must be to have $A_{n+1}=A_{n+1}^{\prime \prime}$. The way out of this is of course a (straightforward) fixed point argument that yields a self consistent $\mu_{n+1} \approx L^{2} \mu_{n}$. See [14, Lemmas 6.2 and 6.6].

So far we have skirted the issue of bounding the perturbative correction $p_{n}$ in our main result. To measure the size of $p_{n}$, we introduce a norm whose finiteness implies that all the kernels in its power series representation are small with $\mathfrak{v}_{0}$ and decay exponentially as their arguments separate in $\mathcal{X}_{0}^{(n)}$. For pedagogical simplicity pretend that $p_{n}$ is a function of only two fields - $\psi$ and one derivative field $\psi_{\nu}$. It has a power series expansion

$$
p_{n}\left(\psi, \psi_{\nu}\right)=\sum_{\substack{r, s \in \mathbb{N}_{0} \\ r+s>0}} \sum_{\substack{\mathbf{x} \in\left(\mathcal{X}_{0}^{(n)}\right)^{r} \\ \mathbf{y} \in\left(\mathcal{X}_{0}^{(n)}\right)^{s}}} p_{n r s}(\mathbf{x}, \mathbf{y}) \psi(\mathbf{x}) \psi_{\nu}(\mathbf{y})
$$

with the notations, $\mathbb{N}_{0}=\mathbb{N} \cup\{0\}$, and

$$
\psi(\mathbf{x})=\psi\left(x_{1}\right) \ldots \psi\left(x_{r}\right)
$$

Each $p_{n r s}(\mathbf{x}, \mathbf{y})$ is separately invariant under permutations of the components of $\mathbf{x}$ and under permutations of the components of $\mathbf{y}$. The norm of $p_{n}$ is

$$
\left\|p_{n}\right\|^{(n)}=\sum_{\substack{r, s \in \mathbb{N}_{0} \\ r+s>0}}\left\|p_{n r s}\right\|_{m} \kappa_{n}^{r} \kappa_{n}^{\prime s} .
$$

For a translation invariant kernel with four arguments, like the interaction kernel $V_{0}\left(x_{1}, x_{2}, x_{3}, x_{4}\right),\left\|V_{0}\right\|_{m}$ is the (mass $m$ ) exponentially weighted $L^{1}-$ $L^{\infty}$ norm of $V_{0}$ :

$$
\left\|V_{0}\right\|_{m}=\max _{j=1,2,3,4} \sup _{x_{j} \in \mathcal{X}_{0}} \sum_{\substack{x_{k} \in \mathcal{X}_{0} \\ k \neq j}}\left|V_{0}\left(x_{1}, x_{2}, x_{3}, x_{4}\right)\right| e^{m \tau\left(x_{1}, x_{2}, x_{3}, x_{4}\right)}
$$

where $\tau\left(x_{1}, x_{2}, x_{3}, x_{4}\right)$ is the minimal length of a tree graph in $\mathcal{X}_{0}$ that has $x_{1}, x_{2}, x_{3}, x_{4}$ among its vertices, and $m \geq 0$ is a fixed decay rate. (The small 'coupling constant' $\mathfrak{v}_{0}=2\left\|V_{0}\right\|_{2 m}$.) The norm $\|w\|_{m}$ of a kernel $w$ with an arbitrary number of arguments is defined in much the same way. For details see $[13, \S 1.4$ and Definition A.3].

Ideally, $\left\|p_{n}\right\|^{(n)}$ would be bounded (and in fact small) uniformly in $n$. Unfortunately, such a bound is too naive to achieve the upper limit on $n$ stated in our main result. The reason is that while the coefficient of an irrelevant monomial decreases as the scale $n$ increases, the maximum allowed size of fields in the domain $S_{n}$ also increases, so the monomial as a whole can be relatively large. So we have chosen

- to move all quartic $\left(\psi_{*} \psi\right)^{2}$ monomials out of $p_{n}$ into $A_{n}$, i.e., to also renormalize the interaction $V_{n}$, and

- to split $p_{n}$ into two parts, 
○ one, called $\mathcal{E}_{n}\left(\psi_{*}, \psi\right)$, is an analytic function whose size is measured in terms of a norm-like $\|\cdot\|^{(n)}$ and is small (and decreasing with $n$ ) and

$\circ$ the other, called $\mathcal{R}_{n}$, is a polynomial of fixed degree, the size of whose coefficient kernels are measured in terms of a norm-like $\|\cdot\|_{m}$.

The details are stated in our main result, [13, Theorem 1.17].

\section{Appendix A. Seeing the Parabolic and Elliptic Regimes}

In this appendix, we perform several model computations that contrast the parabolic nature of the early renormalization group steps with the elliptical nature of the late renormalization group steps. We imagine that after $n$ (block spin) renormalization group steps we have an action whose dominant part (that we are simplifying a bit ${ }^{18}$ ) is $A_{n}\left(\psi_{*}, \psi, \phi_{* n}\left(\psi_{*}, \psi\right), \phi_{n}\left(\psi_{*}, \psi\right)\right)$ where

$$
\begin{aligned}
A_{n}\left(\psi_{*}, \psi, \phi_{*}, \phi\right)= & \left\langle\left(\psi_{*}-Q_{n} \phi_{*}\right),\left(\psi-Q_{n} \phi\right)\right\rangle_{0}+\left\langle\phi_{*},\left(-d_{n} \partial_{0}-\Delta\right) \phi\right\rangle_{n} \\
& -\mu_{n}\left\langle\phi_{*}, \phi\right\rangle_{n}+\frac{\mathrm{v}_{n}}{2}\left\langle\phi_{*} \phi, \phi_{*} \phi\right\rangle_{n} .
\end{aligned}
$$

Here

$\circ\langle f, g\rangle_{0}=\sum_{x \in \mathcal{Y}_{0}} f(x) g(x)$ and $\langle f, g\rangle_{n}=\tilde{\varepsilon}_{n} \varepsilon_{n}^{3} \sum_{u \in \mathcal{Y}_{n}} f(u) g(u)$ are the natural real inner products on $\mathbb{C}^{\mathcal{Y}_{0}}$ and $\mathbb{C}^{\mathcal{Y}_{n}}$, where the fine lattice ${ }^{19} \mathcal{Y}_{n}$ is a finite periodic box in $\tilde{\varepsilon}_{n} \mathbb{Z} \times \varepsilon_{n} \mathbb{Z}^{3}$ (the lattice spacings $\tilde{\varepsilon}_{n}$ and $\varepsilon_{n}$ are small) and the unit lattice $\mathcal{Y}_{0}$ is a finite periodic box in $\mathbb{Z} \times \mathbb{Z}^{3}$ and is a sublattice of $\mathcal{Y}_{n}$.

- $Q_{n}: \mathbb{C}^{\mathcal{Y}_{n}} \rightarrow \mathbb{C}^{\mathcal{Y}_{0}}$ is the linear map for which $\left(Q_{n} f\right)(x)$ is the average of $f \in \mathbb{C}^{\mathcal{Y}_{n}}$ over the square box in $\mathcal{Y}_{n}$ centered at $x \in \mathcal{Y}_{0}$ with sides 1. This box contains $\frac{1}{\tilde{\varepsilon}_{n} \varepsilon_{n}^{3}}$ points of $\mathcal{Y}_{n}$.

- $\partial_{0}$ and $\Delta$ are the discrete forward time derivative and Laplacian on $\mathcal{Y}_{n}$, respectively.

- $\mu_{n}>0$ is the renormalized chemical potential. It is small in the parabolic regime and large in the elliptic regime. $\mathrm{v}_{n}>0$ is the renormalized coupling constant. It is small. $d_{n}>0$ is one in the parabolic regime and large in the elliptic regime.

○ For each $\psi_{*}, \psi \in \mathbb{C}^{\mathcal{Y}_{0}}$ the fields $\phi_{* n}\left(\psi_{*}, \psi\right), \phi_{n}\left(\psi_{*}, \psi\right)$ on $\mathcal{Y}_{n}$ are critical points of the functional

$$
\left(\phi_{*}, \phi\right) \mapsto A_{n}\left(\psi_{*}, \psi, \phi_{*}, \phi\right)
$$

\footnotetext{
18 In particular, for pedagogical purposes, we have replaced $a_{n}$ by 1 and replaced $\mathcal{V}_{n}$ by a local interaction.

19 The fine lattice $\mathcal{Y}_{n}$ is a rescaled version of the original lattice $\mathcal{X}_{0}$ of (1).
} 
They obey the background field equations

$$
\begin{aligned}
\frac{\delta}{\delta \phi_{*}} A_{n}\left(\psi_{*}, \psi, \phi_{*}, \phi\right) & =Q_{n}^{*}\left(Q_{n} \phi-\psi\right)+D_{n} \phi+\left(\mathrm{v}_{n} \phi_{*} \phi-\mu_{n}\right) \phi=0 \\
\frac{\delta}{\delta \phi} A_{n}\left(\psi_{*}, \psi, \phi_{*}, \phi\right) & =Q_{n}^{*}\left(Q_{n} \phi_{*}-\psi_{*}\right)+D_{n}^{*} \phi_{*}+\left(\mathrm{v}_{n} \phi_{*} \phi-\mu_{n}\right) \phi_{*}=0
\end{aligned}
$$

with $D_{n}=-d_{n} \partial_{0}-\Delta$.

\section{A.1. Constant Field Background Fields}

To start getting a feel for the background field equations (A.2), we consider the case that $\psi_{*}$ and $\psi$ are constant fields with $\psi_{*}=\psi^{*}$. We'll look for solutions $\phi_{(*)}$ which are also constant fields with $\phi_{*}=\phi^{*}$. Since both $Q_{n}$ and $Q_{n}^{*}$ map the constant function 1 to the constant function 1, the constant field background fields obey

$$
\phi+\left(\mathrm{v}_{n}|\phi|^{2}-\mu_{n}\right) \phi=\psi .
$$

This is of the form 'real number times $\phi$ equals real number times $\psi$ ' so the phase of $\phi$ and $\psi$ will be the same (modulo $\pi$ ). So it suffices to consider the case that $\psi$ and $\phi$ are both real and obey

$$
\phi+\left(\mathrm{v}_{n} \phi^{2}-\mu_{n}\right) \phi=\psi .
$$

Since

$$
\frac{d}{d \phi}\left[\phi+\left(\mathrm{v}_{n} \phi^{2}-\mu_{n}\right) \phi\right]=1-\mu_{n}+3 \mathrm{v}_{n} \phi^{2} \begin{cases}\geq 0 & \text { if } \mu_{n} \leq 1 \\ >0 & \text { if } \mu_{n}>1,|\phi|>\sqrt{\frac{\mu_{n}-1}{3 \mathrm{v}_{n}}} \\ <0 & \text { if } \mu_{n}>1,|\phi|<\sqrt{\frac{\mu_{n}-1}{3 \mathrm{v}_{n}}}\end{cases}
$$

there is always exactly one solution when $\mu_{n} \leq 1$, but the solution can be nonunique when $\mu_{n}>1$. For example, when $\mu_{n}>1$ and $\psi=0$, the solutions are $\phi=0$ and $\phi= \pm \sqrt{\frac{\mu_{n}-1}{\mathrm{v}_{n}}}$.

\section{A.2. The Background Field in the Parabolic Regime}

Imagine that we wish to solve the background field equations (A.2) for $\phi_{(*)}$ as analytic functions of $\psi_{(*)}$, in the parabolic regime, when $\mu_{n}$ is small, so that the minimum of the effective potential is still near the origin-see (5). Then

$$
\begin{aligned}
\left(Q_{n}^{*} Q_{n}+D_{n}-\mu_{n}\right) \phi & =Q_{n}^{*} \psi-\mathrm{v}_{n} \phi_{*} \phi^{2} \\
\left(Q_{n}^{*} Q_{n}+D_{n}^{*}-\mu_{n}\right) \phi_{*} & =Q_{n}^{*} \psi_{*}-\mathrm{v}_{n} \phi_{*}^{2} \phi
\end{aligned}
$$

and, to first order in $\psi_{(*)}$,

$$
\begin{aligned}
\phi & =\left(Q_{n}^{*} Q_{n}-\mu_{n}-d_{n} \partial_{0}-\Delta\right)^{-1} Q_{n}^{*} \psi+O\left(\psi_{(*)}^{3}\right) \\
\phi_{*} & =\left(Q_{n}^{*} Q_{n}-\mu_{n}-d_{n} \partial_{0}^{*}-\Delta\right)^{-1} Q_{n}^{*} \psi_{*}+O\left(\psi_{(*)}^{3}\right) .
\end{aligned}
$$

We are interested in small $\psi_{(*)}$, so the $O\left(\psi_{(*)}^{3}\right)$ corrections are unimportant.

We here see the parabolic (discrete) differential operators $d_{n} \partial_{0}^{(*)}+\Delta$. 


\section{A.3. The Background Field in the Elliptic Regime}

Imagine that we again wish to solve the background field equations (A.2), but this time in the elliptic regime when $\mu_{n}$ is large, $\mathrm{v}_{n}$ is small and the effective potential has a deep well, whose minima form a circle in the complex plane of radius $r_{n}=\sqrt{\frac{\mu_{n}}{\mathrm{v}_{n}}}$. We are interested in $\psi_{(*)}$ and $\phi_{(*)}$ near the minimum of the effective potential. That is, with $\left|\psi_{(*)}\right|,\left|\phi_{(*)}\right| \approx r_{n}$. We write

$$
\psi=r_{n} e^{R+i \Theta} \quad \psi_{*}=r_{n} e^{R-i \Theta} \quad \phi=r_{n} e^{X+i H} \quad \phi_{*}=r_{n} e^{X-i H}
$$

and look for solutions when $R, \Theta$ are small. Substitute into (A.2) and divide by $r_{n}$. This gives

$$
\begin{aligned}
& D_{n}\left[e^{X+i H}\right]+Q_{n}^{*}\left(Q_{n} e^{X+i H}-e^{R+i \Theta}\right)+\mu_{n}\left(e^{2 X}-1\right) e^{X+i H}=0 \\
& D_{n}^{*}\left[e^{X-i H}\right]+Q_{n}^{*}\left(Q_{n} e^{X-i H}-e^{R-i \Theta}\right)+\mu_{n}\left(e^{2 X}-1\right) e^{X-i H}=0 .
\end{aligned}
$$

Expand the exponentials, keeping only terms to first order in $\{R, \Theta, X, H\}$, to get

$$
\begin{aligned}
& D_{n}(X+i H)+Q_{n}^{*} Q_{n}(X+i H)+2 \mu_{n} X=Q_{n}^{*}(R+i \Theta) \\
& D_{n}^{*}(X-i H)+Q_{n}^{*} Q_{n}(X-i H)+2 \mu_{n} X=Q_{n}^{*}(R-i \Theta) .
\end{aligned}
$$

Now simplify, by adding together the two equations of (A.5) and dividing by 2 , and then subtracting the second equation of (A.5) from the first and dividing by $2 i$. Pretend that $\partial_{0}$ is a continuum partial derivative rather than a discrete forward derivative. Then

$$
\begin{aligned}
\frac{1}{2}\left(D_{n}+D_{n}^{*}\right) & =-\frac{d_{n}}{2}\left(\partial_{0}+\partial_{0}^{*}\right)-\Delta=-\Delta \\
\frac{1}{2 i}\left(D_{n}-D_{n}^{*}\right) & =\frac{i}{2} d_{n}\left(\partial_{0}-\partial_{0}^{*}\right)=i d_{n} \partial_{0}
\end{aligned}
$$

and (A.5) gives

$$
\begin{aligned}
{\left[2 \mu_{n}-\Delta+Q_{n}^{*} Q_{n}\right] X-i d_{n} \partial_{0} H } & =Q_{n}^{*} R \\
i d_{n} \partial_{0} X+\left[-\Delta+Q_{n}^{*} Q_{n}\right] H & =Q_{n}^{*} \Theta
\end{aligned}
$$

or, in matrix form,

$$
\square\left[\begin{array}{l}
X \\
H
\end{array}\right]=Q_{n}^{*}\left[\begin{array}{l}
R \\
\Theta
\end{array}\right] \quad \text { or } \quad\left[\begin{array}{c}
X \\
H
\end{array}\right]=\square^{-1} Q_{n}^{*}\left[\begin{array}{c}
R \\
\Theta
\end{array}\right]
$$

where

$$
\square=\left[\begin{array}{cc}
2 \mu_{n}-\Delta & i d_{n} \partial_{0}^{*} \\
i d_{n} \partial_{0} & -\Delta
\end{array}\right]+Q_{n}^{*} Q_{n}
$$

The $Q_{n}^{*} Q_{n}$ provides a mass which makes $\square$ boundedly invertible. But, the presence of this mass is a consequence of our having rescaled the original unit lattice down to the very fine lattice $\mathcal{Y}_{n}$. To invert $\square$, ignoring the $Q_{n}^{*} Q_{n}$, we have to divide, essentially, by

$$
\operatorname{det}\left[\begin{array}{cc}
2 \mu_{n}-\Delta & i d_{n} \partial_{0}^{*} \\
i d_{n} \partial_{0} & -\Delta
\end{array}\right]=d_{n}^{2}\left\{\partial_{0}^{*} \partial_{0}+2 \frac{\mu_{n}}{d_{n}^{2}}(-\Delta)+\frac{1}{d_{n}^{2}}(-\Delta)^{2}\right\} \text {. }
$$


- In the parabolic regime, $\mu_{n}$ is small and $d_{n}$ is essentially one so that the operator in the curly brackets is approximately $\partial_{0}^{*} \partial_{0}+(-\Delta)^{2}$, which is parabolic.

- In the elliptic regime, $\mu_{n}$ and $d_{n}$ are both very large with $\frac{\mu_{n}}{d_{n}^{2}}>0$ being essentially independent of $n$. So the operator in the curly brackets is approximately $\partial_{0}^{*} \partial_{0}++2 \frac{\mu_{n}}{d_{n}^{2}}(-\Delta)$, which is elliptic.

\section{A.4. The Quadratic Approximation to the Action}

For the remaining model computations, we study the quadratic approximation to the action (A.1).

A.4.a. Expanding Around Zero Field. We first consider the parabolic regime as studied in $[13,14]$. Substitute the linear approximation to the background fields $\phi_{(*)}$ (as functions on $\psi_{(*)}$ ) of (A.3) into the action (A.1), keeping only terms that are of degree at most two in $\psi_{(*)}$. Writing

$$
S_{n}\left(\mu_{n}\right)=\left(Q_{n}^{*} Q_{n}-\mu_{n}+D_{n}\right)^{-1}
$$

(A.3) becomes

$$
\phi=S_{n}\left(\mu_{n}\right) Q_{n}^{*} \psi+O\left(\psi_{(*)}^{3}\right) \quad \phi_{*}=S_{n}\left(\mu_{n}\right)^{*} Q_{n}^{*} \psi_{*}+O\left(\psi_{(*)}^{3}\right)
$$

so that

$$
\begin{aligned}
A_{n}= & \left\langle\left(\psi_{*}-Q_{n} \phi_{*}\right),\left(\psi-Q_{n} \phi\right)\right\rangle_{0}+\left\langle\phi_{*},\left(D_{n}-\mu_{n}\right) \phi\right\rangle_{n}+O\left(\psi_{(*)}^{4}\right) \\
= & \left\langle\psi_{*}, \psi\right\rangle_{0}-\left\langle\psi_{*}, Q_{n} \phi\right\rangle_{0}-\left\langle Q_{n} \phi_{*}, \psi\right\rangle_{0}+\left\langle\phi_{*},\left(Q_{n}^{*} Q_{n}+D_{n}-\mu_{n}\right) \phi\right\rangle_{n} \\
& +O\left(\psi_{(*)}^{4}\right) \\
= & \left\langle\psi_{*}, \psi\right\rangle_{0}-\left\langle\psi_{*}, Q_{n} S_{n}\left(\mu_{n}\right) Q_{n}^{*} \psi\right\rangle_{0}-\left\langle Q_{n} S_{n}\left(\mu_{n}\right)^{*} Q_{n}^{*} \psi_{*}, \psi\right\rangle_{0} \\
& +\left\langle S_{n}\left(\mu_{n}\right)^{*} Q_{n}^{*} \psi_{*}, Q_{n}^{*} \psi\right\rangle_{n}+O\left(\psi_{(*)}^{4}\right) \\
= & \left\langle\psi_{*},\left(\mathbb{1}-Q_{n} S_{n}\left(\mu_{n}\right) Q_{n}^{*}\right) \psi\right\rangle_{0}+O\left(\psi_{(*)}^{4}\right) .
\end{aligned}
$$

We now analyze the operator $\mathbb{1}-Q_{n} S_{n}\left(\mu_{n}\right) Q_{n}^{*}$ in momentum space, in the special case that $\mu_{n}=0$, and see that it is basically a (discrete) parabolic differential operator. Set

$$
\Delta^{(n)}=\left(\mathbb{1}+Q_{n} D_{n}^{-1} Q_{n}^{*}\right)^{-1} .
$$

Substituting in the definitions and simplifying, we see that

$$
S_{n}(0)^{-1} D_{n}^{-1} Q_{n}^{*} \Delta^{(n)}=Q_{n}^{*}
$$

so that

$$
Q_{n} S_{n}(0) Q_{n}^{*}=Q_{n} D_{n}^{-1} Q_{n}^{*} \Delta^{(n)} .
$$

By [10, Remark 2.1.e], with $\mathfrak{q}=1$,

$$
\widehat{\left(Q_{n} \phi\right)}(k)=\sum_{\ell \in \hat{\mathcal{B}}_{n}} u_{n}(k+\ell) \hat{\phi}(k+\ell) \quad \widehat{\left(Q_{n}^{*} \psi\right)}(k+\ell)=u_{n}(k+\ell) \hat{\psi}(k)
$$


where

$$
u_{n}(p)=\frac{\sin \left(\frac{1}{2} p_{0}\right)}{\frac{1}{\tilde{\varepsilon}_{n}} \sin \left(\frac{1}{2} \tilde{\varepsilon}_{n} p_{0}\right)} \prod_{\nu=1}^{3} \frac{\sin \left(\frac{1}{2} p_{\nu}\right)}{\frac{1}{\varepsilon_{n}} \sin \left(\frac{1}{2} \varepsilon_{n} p_{\nu}\right)} .
$$

Here $k$ runs over the dual lattice of $\mathcal{Y}_{0}$ and $k+\ell$ runs over the dual lattice of $\mathcal{Y}_{n}$. We do not need to know much about these dual lattices, except that the dual lattice of $\mathcal{Y}_{0}$ is a discretization of $(\mathbb{R} / 2 \pi \mathbb{Z}) \times\left(\mathbb{R}^{3} / 2 \pi \mathbb{Z}^{3}\right)$, the dual lattice of $\mathcal{Y}_{n}$ is a discretization of $\left(\mathbb{R} / \frac{2 \pi}{\tilde{\varepsilon}_{n}} \mathbb{Z}\right) \times\left(\mathbb{R}^{3} / \frac{2 \pi}{\varepsilon_{n}} \mathbb{Z}^{3}\right)$, and $\ell$ runs over

$$
\hat{\mathcal{B}}_{n}=\left(2 \pi \mathbb{Z} / \frac{2 \pi}{\tilde{\varepsilon}_{n}} \mathbb{Z}\right) \times\left(2 \pi \mathbb{Z}^{3} / \frac{2 \pi}{\varepsilon_{n}} \mathbb{Z}^{3}\right) \text {. }
$$

So, by [10, Lemmas 2.2.b,c, 3.2.d and 4.2.b, and Remark 4.1.a], with $\mathfrak{q}=1$ and $\mathfrak{Q}_{n}$ replaced by $\mathbb{1}$, the operator $Q_{n} S_{n}(0) Q_{n}^{*}$ has Fourier transform

$$
\begin{aligned}
& \sum_{\ell \in \hat{\mathcal{B}}_{n}} u_{n}(k+\ell) \hat{D}_{n}^{-1}(k+\ell) u_{n}(k+\ell) \hat{\Delta}^{(n)}(k) \\
& \quad=u_{n}(k)^{2} \hat{D}_{n}^{-1}(k) \hat{\Delta}^{(n)}(k)+\sum_{0 \neq \ell \in \hat{\mathcal{B}}_{n}} u_{n}(k+\ell)^{2} \hat{D}_{n}^{-1}(k+\ell) \hat{\Delta}^{(n)}(k) \\
& =\frac{u_{n}(k)^{2}}{u_{n}(k)^{2}+\hat{D}_{n}(k)+O\left(|k|^{3}\right)}+\sum_{0 \neq \ell \in \hat{\mathcal{B}}_{n}} O\left(|k|^{2}\right) \prod_{\nu=0}^{3}\left[\frac{24}{\left|\ell_{\nu}\right|+\pi}\right]^{2} O(1) O(|k|) \\
& =\frac{1}{1+\hat{D}_{n}(k) u_{n}(k)^{-2}+O\left(|k|^{3}\right)}+\sum_{0 \neq \ell \in \hat{\mathcal{B}}_{n}} O\left(|k|^{3}\right) \prod_{\nu=0}^{3}\left[\frac{24}{\left|\ell_{\nu}\right|+\pi}\right]^{2} \\
& =\frac{1}{1+\hat{D}_{n}(k)+O\left(|k|^{3}\right)}+O\left(|k|^{3}\right)
\end{aligned}
$$

and $\mathbb{1}-Q_{n} S_{n}(0) Q_{n}^{*}$ has Fourier transform

$$
\begin{aligned}
1-\frac{1}{1+\hat{D}_{n}(k)+O\left(|k|^{3}\right)}+O\left(|k|^{3}\right) & =\hat{D}_{n}(k)+O\left(\hat{D}_{n}(k)^{2}\right)+O\left(|k|^{3}\right) \\
& =-i d_{n} k_{0}+\mathbf{k}^{2}+O\left(k_{0}^{2}\right)+O\left(|k|^{3}\right)
\end{aligned}
$$

and so is a parabolic operator.

A.4.b. Expanding Around the Bottom of the Effective Potential. For all $\mu_{n} \neq$ 0 , it is appropriate to expand the action about the bottom of the effective potential, rather than about the origin. That is, rather than in powers of $\psi_{(*)}$. So we rewrite the action (A.1)

$$
\begin{aligned}
A_{n}= & \left\langle\left(\psi_{*}-Q_{n} \phi_{*}\right),\left(\psi-Q_{n} \phi\right)\right\rangle_{0}+\left\langle\phi_{*},\left(-d_{n} \partial_{0}-\Delta\right) \phi\right\rangle_{n} \\
& +\frac{\mathrm{v}_{n}}{2}\left\langle\left[\phi_{*} \phi-r_{n}^{2}\right]^{2}, 1\right\rangle_{n}-\frac{r_{n}^{4} \mathrm{v}_{n}}{2}\langle 1,1\rangle_{n}
\end{aligned}
$$

and then substitute the representations (A.4) of $\psi_{(*)}$ and $\phi_{(*)}$ in terms of radial and tangential fields. Note that when $R=\Theta=X=H=0$, the field magnitudes $\left|\psi_{(*)}\right|=\left|\phi_{(*)}\right|=r_{n}$ and $\psi_{(*)}$ and $\phi_{(*)}$ are at the bottom of the effective potential. Still pretending that $\partial_{0}$ is a continuous derivative, and using 
the notation $O[3]=O\left(X^{3}+R^{3}+H^{3}+\Theta^{3}\right)$, we get the following representation of the action, which is reminiscent of (A.8).

\section{Lemma A.1.}

$$
\frac{1}{r_{n}^{2}} A_{n}=\left\langle\left[\begin{array}{c}
R \\
\Theta
\end{array}\right],\left\{\mathbb{1}-Q_{n} \square^{-1} Q_{n}^{*}\right\}\left[\begin{array}{c}
R \\
\Theta
\end{array}\right]\right\rangle_{0}-\frac{r_{n}^{2} \mathrm{v}_{n}}{2}\langle 1,1\rangle_{n}+O[3] .
$$

Proof. The three main terms in $A_{n}$ are

$$
\begin{aligned}
\left\langle\left(\psi_{*}\right.\right. & \left.\left.-Q_{n} \phi_{*}\right),\left(\psi-Q_{n} \phi\right)\right\rangle_{0} \\
= & r_{n}^{2}\left\langle\left(e^{R-i \Theta}-Q_{n} e^{X-i H}\right),\left(e^{R+i \Theta}-Q_{n} e^{X+i H}\right)\right\rangle_{0} \\
= & r_{n}^{2}\left\langle R-i \Theta-Q_{n}(X-i H), R+i \Theta-Q_{n}(X+i H)\right\rangle_{0}+O[3] \\
= & r_{n}^{2}\left\langle\left[\begin{array}{l}
R \\
\Theta
\end{array}\right],\left[\begin{array}{l}
R \\
\Theta
\end{array}\right]\right\rangle_{0}-2 r_{n}^{2}\left\langle\left[\begin{array}{l}
R \\
\Theta
\end{array}\right], Q_{n}\left[\begin{array}{l}
X \\
H
\end{array}\right]\right\rangle_{0}+r_{n}^{2}\left\langle\left[\begin{array}{l}
X \\
H
\end{array}\right], Q_{n}^{*} Q_{n}\left[\begin{array}{l}
X \\
H
\end{array}\right]\right\rangle_{n} \\
& +O[3]
\end{aligned}
$$

and

$$
\begin{aligned}
\left\langle\phi_{*},\left(-d_{n} \partial_{0}-\Delta\right) \phi\right\rangle_{n} & =r_{n}^{2}\left\langle e^{X-i H},\left(-d_{n} \partial_{0}-\Delta\right) e^{X+i H}\right\rangle_{n} \\
& =r_{n}^{2}\left\langle X-i H,\left(-d_{n} \partial_{0}-\Delta\right)(X+i H)\right\rangle_{n}+O[3] \\
& =r_{n}^{2}\left\langle\left[\begin{array}{l}
X \\
H
\end{array}\right],\left[\begin{array}{cc}
-\Delta & i d_{n} \partial_{0}^{*} \\
i d_{n} \partial_{0} & -\Delta
\end{array}\right]\left[\begin{array}{l}
X \\
H
\end{array}\right]\right\rangle+O[3]
\end{aligned}
$$

and

$$
\begin{aligned}
\frac{\mathrm{v}_{n}}{2}\left\langle\left[\phi_{*} \phi-r_{n}^{2}\right]^{2}, 1\right\rangle_{n} & =\frac{\mathrm{v}_{n} r_{n}^{4}}{2}\left\langle\left[e^{2 X}-1\right]^{2}, 1\right\rangle_{n} \\
& =2 r_{n}^{2} \mu_{n}\langle X, X\rangle_{n}+O[3] \\
& =r_{n}^{2}\left\langle\left[\begin{array}{l}
X \\
H
\end{array}\right],\left[\begin{array}{rr}
2 \mu_{n} & 0 \\
0 & 0
\end{array}\right]\left[\begin{array}{l}
X \\
H
\end{array}\right]\right\rangle+O[3]
\end{aligned}
$$

So all together

$$
\begin{aligned}
\frac{1}{r_{n}^{2}} A_{n}= & \left\langle\left[\begin{array}{l}
R \\
\Theta
\end{array}\right],\left[\begin{array}{l}
R \\
\Theta
\end{array}\right]\right\rangle_{0}-2\left\langle\left[\begin{array}{l}
R \\
\Theta
\end{array}\right], Q_{n}\left[\begin{array}{l}
X \\
H
\end{array}\right]\right\rangle_{0} \\
& +\left\langle\left[\begin{array}{l}
X \\
H
\end{array}\right],\left\{\left[\begin{array}{cc}
2 \mu_{n}-\Delta i d_{n} \partial_{0}^{*} \\
i d_{n} \partial_{0} & -\Delta
\end{array}\right]+Q_{n}^{*} Q_{n}\right\}\left[\begin{array}{l}
X \\
H
\end{array}\right]\right\rangle_{n} \\
& -\frac{r_{n}^{2} v_{n}}{2}\langle 1,1\rangle_{n}+O[3] \\
= & \left\langle\left[\begin{array}{l}
R \\
\Theta
\end{array}\right],\left[\begin{array}{l}
R \\
\Theta
\end{array}\right]\right\rangle_{0}-2\left\langle\left[\begin{array}{l}
R \\
\Theta
\end{array}\right], Q_{n}\left[\begin{array}{l}
X \\
H
\end{array}\right]\right\rangle_{0}+\left\langle\left[\begin{array}{l}
X \\
H
\end{array}\right], \square\left[\begin{array}{l}
X \\
H
\end{array}\right]\right\rangle_{n} \\
& -\frac{r_{n}^{2} \mathrm{v}_{n}}{2}\langle 1,1\rangle_{n}+O[3] .
\end{aligned}
$$


Substituting in (A.6), we have

$$
\begin{aligned}
\frac{1}{r_{n}^{2}} A_{n}= & \left\langle\left[\begin{array}{l}
R \\
\Theta
\end{array}\right],\left[\begin{array}{l}
R \\
\Theta
\end{array}\right]\right\rangle_{0}-2\left\langle\left[\begin{array}{l}
R \\
\Theta
\end{array}\right], Q_{n} \square^{-1} Q_{n}^{*}\left[\begin{array}{l}
R \\
\Theta
\end{array}\right]\right\rangle_{0} \\
& +\left\langle\square^{-1} Q_{n}^{*}\left[\begin{array}{c}
R \\
\Theta
\end{array}\right], \square^{-1} Q_{n}^{*}\left[\begin{array}{l}
R \\
\Theta
\end{array}\right]\right\rangle_{n}-\frac{r_{n}^{2} \mathrm{v}_{n}}{2}\langle 1,1\rangle_{n}+O[3] \\
= & \left\langle\left[\begin{array}{l}
R \\
\Theta
\end{array}\right],\left\{11-Q_{n} \square^{-1} Q_{n}^{*}\right\}\left[\begin{array}{c}
R \\
\Theta
\end{array}\right]\right\rangle_{0}-\frac{r_{n}^{2} \mathrm{v}_{n}}{2}\langle 1,1\rangle_{n}+O[3]
\end{aligned}
$$

as desired.

So now we should analyze the operator $\mathbb{1}-Q_{n} \square^{-1} Q_{n}^{*}$ in momentum space. We follow the pattern of the computation from (A.9) through (A.11). Define

$$
\mathbb{D}_{n}=\left[\begin{array}{cc}
2 \mu_{n}-\Delta & i d_{n} \partial_{0}^{*} \\
i d_{n} \partial_{0} & -\Delta
\end{array}\right]
$$

and, analogously to (A.9),

$$
\tilde{\mathbb{D}}_{n}=\left(\mathbb{1}+Q_{n} \mathbb{D}_{n}^{-1} Q_{n}^{*}\right)^{-1} .
$$

Substituting these definitions into $\square \mathbb{D}_{n}^{-1} Q_{n}^{*} \tilde{\mathbb{D}}_{n}$ and simplifying yields $Q_{n}^{*}$ so that

$$
Q_{n} \square^{-1} Q_{n}^{*}=Q_{n} \mathbb{D}_{n}^{-1} Q_{n}^{*} \tilde{\mathbb{D}}_{n}
$$

The Fourier transform of $Q_{n} \square^{-1} Q_{n}^{*}$ is

$$
\begin{aligned}
& \sum_{\ell \in \hat{\mathcal{B}}_{n}} u_{n}(k+\ell) \hat{\mathbb{D}}_{n}^{-1}(k+\ell) u_{n}(k+\ell) \widehat{\tilde{\mathbb{D}}}_{n}(k) \\
& \quad=u_{n}(k)^{2} \hat{\mathbb{D}}_{n}^{-1}(k) \widehat{\tilde{\mathbb{D}}}_{n}(k)+\sum_{0 \neq \ell \in \hat{\mathcal{B}}_{n}} u_{n}(k+\ell)^{2} \hat{\mathbb{D}}_{n}^{-1}(k+\ell) \widehat{\tilde{\mathbb{D}}}_{n}(k)
\end{aligned}
$$

where, pretending that we have continuum, rather than discrete, differential operators,

$$
\hat{\mathbb{D}}_{n}(p)=\left[\begin{array}{cc}
2 \mu_{n}+\mathbf{p}^{2} & d_{n} p_{0} \\
-d_{n} p_{0} & \mathbf{p}^{2}
\end{array}\right]
$$

and

$$
\widehat{\tilde{\mathbb{D}}}_{n}(k)=\left(\mathbb{1}+\sum_{\ell \in \hat{\mathcal{B}}_{n}} u_{n}(k+\ell)^{2} \hat{\mathbb{D}}_{n}^{-1}(k+\ell)\right)^{-1}
$$

During the course of the upcoming computation, we shall use the following facts.

- The parameter $d_{n} \geq 1$. For small $n$, it takes the value 1 and for large $n$, it decays quickly approaching 0 as $n \rightarrow \infty$.

- The parameter $\mu_{n}>0$. For small $n$, it is very small and for large $n$, it is very large, with $d_{n}^{-2} \mu_{n}$ bounded uniformly in $n$. When $d_{n}>1, d_{n}^{-2} \mu_{n}$ is bounded away from zero. 
- By [10, Lemma 2.2.b,c].

$$
\begin{aligned}
& \circ u_{n}(k)=1+O\left(|k|^{2}\right) \text { and } \\
& \circ \text { if } \ell \neq 0,\left|u_{n}(k+\ell)\right| \leq\left[\prod_{\substack{0 \leq \nu \leq 3 \\
\ell_{\nu} \neq 0}}\left|k_{\nu}\right|\right] \prod_{\nu=0}^{3} \frac{24}{\left|\ell_{\nu}\right|+\pi} .
\end{aligned}
$$

The dominant term in (A.13) is

$$
\begin{aligned}
u_{n}(k)^{2} \hat{\mathbb{D}}_{n}^{-1}(k) \widehat{\tilde{\mathbb{D}}}_{n}(k)=u_{n}(k)^{2} \hat{\mathbb{D}}_{n}^{-1}(k)\left\{\mathbb{1}+\sum_{\ell \in \hat{\mathcal{B}}_{n}} u_{n}(k+\ell)^{2} \hat{\mathbb{D}}_{n}^{-1}(k+\ell)\right\}^{-1} \\
\quad=\left\{\mathbb{1}+\hat{\mathbb{D}}_{n}(k) u_{n}(k)^{-2}+\sum_{0 \neq \ell \in \hat{\mathcal{B}}_{n}} \frac{u_{n}(k+\ell)^{2}}{u_{n}(k)^{2}} \hat{\mathbb{D}}_{n}^{-1}(k+\ell) \hat{\mathbb{D}}_{n}(k)\right\}^{-1} \\
=\left\{\mathbb{1}+\hat{\mathbb{D}}_{n}(k) u_{n}(k)^{-2}+O\left(|k|^{2}\left[\begin{array}{cc}
d_{n}^{-2} \mu_{n}+|k| & d_{n}^{-1}|k| \\
d_{n}^{-1} \mu_{n}+d_{n}|k| & |k|
\end{array}\right]\right\}^{-1}\right.
\end{aligned}
$$

(by Lemma A.2.b)

$$
\begin{aligned}
& =\left[\begin{array}{cc}
1+\frac{2 \mu_{n}}{u_{n}(k)^{2}}+\mathbf{k}^{2}+O\left(d_{n}^{-2} \mu_{n}|k|^{2}\right)+O\left(|k|^{3}\right) \frac{d_{n} k_{0}}{u_{n}(k)^{2}}+O\left(d_{n}^{-1}|k|^{3}\right) \\
-d_{n} k_{0}+O\left(d_{n}^{-1} \mu_{n}|k|^{2}\right)+O\left(d_{n}|k|^{3}\right) & 1+\mathbf{k}^{2}+O\left(|k|^{3}\right)
\end{array}\right]^{-1} \\
& =\left[\begin{array}{cc}
1+2 \mu_{n}\left(1+q_{1}(k)\right)+\mathbf{k}^{2}+O\left(|k|^{3}\right) & d_{n} k_{0}+O\left(d_{n}|k|^{3}\right) \\
-d_{n} k_{0}+O\left(d_{n}^{-1} \mu_{n}|k|^{2}\right)+O\left(d_{n}|k|^{3}\right) & 1+\mathbf{k}^{2}+O\left(|k|^{3}\right)
\end{array}\right]^{-1}
\end{aligned}
$$

with $q_{1}(k)=O\left(|k|^{2}\right)$. The determinant of the matrix to be inverted in the last line of (A.14.a) is

$$
\begin{aligned}
\operatorname{det} & {\left[\begin{array}{cc}
1+2 \mu_{n}\left(1+q_{1}(k)\right)+\mathbf{k}^{2}+O\left(|k|^{3}\right) & d_{n} k_{0}+O\left(d_{n}|k|^{3}\right) \\
-d_{n} k_{0}+O\left(d_{n}^{-1} \mu_{n}|k|^{2}\right)+O\left(d_{n}|k|^{3}\right) & 1+\mathbf{k}^{2}+O\left(|k|^{3}\right)
\end{array}\right] } \\
= & \left.1+2 \mu_{n}\left(1+q_{1}(k)\right)\right)+2\left(1+\mu_{n}\right) \mathbf{k}^{2}+d_{n}^{2} k_{0}^{2} \\
& +O\left(|k|^{3}\right)+O\left(\mu_{n}|k|^{3}\right)+O\left(d_{n}^{2}|k|^{4}\right) \\
= & d_{n}^{2}\left\{d_{n}^{-2}\left[1+2 \mu_{n}\left(1+q_{1}(k)\right)\right]+k_{0}^{2}+2 d_{n}^{-2}\left(1+\mu_{n}\right) \mathbf{k}^{2}+O\left(|k|^{3}\right)\right\} \\
= & d_{n}^{2}\left\{d_{n}^{-2}+2 d_{n}^{-2} \mu_{n}+q_{2}(k)+O\left(|k|^{3}\right)\right\}
\end{aligned}
$$

where

$$
q_{2}(k)=k_{0}^{2}+2 d_{n}^{-2} \mathbf{k}^{2}+2 d_{n}^{-2} \mu_{n}\left(\mathbf{k}^{2}+q_{1}(k)\right)
$$


The tail of (A.13) is, by Lemma A.2.d,

$$
\sum_{0 \neq \ell \in \hat{\mathcal{B}}_{n}} u_{n}(k+\ell)^{2} \hat{\mathbb{D}}_{n}^{-1}(k+\ell) \widehat{\tilde{\mathbb{D}}}_{n}(k)=O\left(|k|^{2}\left[\begin{array}{cc}
d_{n}^{-4} \mu_{n}+d_{n}^{-2}|k| & d_{n}^{-1}|k| \\
d_{n}^{-3} \mu_{n}+d_{n}^{-1}|k| & |k|
\end{array}\right]\right) \text {. }
$$

Combining (A.13) and the three (A.14)'s, we have that the Fourier transform of $Q_{n} \mathbb{D}_{n}^{-1} Q_{n}^{*} \tilde{\mathbb{D}}_{n}=Q_{n} \square^{-1} Q_{n}^{*}$ is

○

$$
\begin{aligned}
d_{n}^{-2} & \left\{d_{n}^{-2}+2 d_{n}^{-2} \mu_{n}+q_{2}(k)+O\left(|k|^{3}\right)\right\}^{-1} \\
& =\frac{d_{n}^{-2}}{d_{n}^{-2}+2 d_{n}^{-2} \mu_{n}}\left\{1-\frac{q_{2}(k)}{d_{n}^{-2}+2 d_{n}^{-2} \mu_{n}}+O\left(|k|^{3}\right)\right\}
\end{aligned}
$$

○ times

$$
\left[\begin{array}{cc}
1+\mathbf{k}^{2}+O\left(|k|^{3}\right) & -d_{n} k_{0}+O\left(d_{n}|k|^{3}\right) \\
d_{n} k_{0}+O\left(d_{n}^{-1} \mu_{n}|k|^{2}\right)+O\left(d_{n}|k|^{3}\right) & 1+2 \mu_{n}\left(1+q_{1}(k)\right)+\mathbf{k}^{2}+O\left(|k|^{3}\right)
\end{array}\right]
$$

$\circ$ plus

$$
O\left(|k|^{2}\left[\begin{array}{cc}
d_{n}^{-4} \mu_{n}+d_{n}^{-2}|k| & d_{n}^{-1}|k| \\
d_{n}^{-3} \mu_{n}+d_{n}^{-1}|k| & |k|
\end{array}\right]\right)
$$

which is

$$
\left[\begin{array}{cc}
1-\frac{2 d_{n}^{-2} \mu_{n}}{d_{n}^{-2}+2 d_{n}^{-2} \mu_{n}}-q_{3}(k)+O\left(\frac{\mu_{n}|k|^{2}}{d_{n}^{4}}\right)+O\left(\frac{|k|^{3}}{d_{n}^{2}}\right) & -\frac{d_{n}^{-1} k_{0}}{d_{n}^{-2}+2 d_{n}^{-2} \mu_{n}}+O\left(\frac{|k|^{3}}{d_{n}}\right) \\
\frac{d_{n}^{-1} k_{0}}{d_{n}^{-2}+2 d_{n}^{-2} \mu_{n}}+O\left(\frac{\mu_{n}|k|^{2}}{d_{n}^{3}}\right)+O\left(\frac{|k|^{3}}{d_{n}}\right) & 1-q_{4}(k)+O\left(|k|^{3}\right)
\end{array}\right]
$$

with

$$
\begin{aligned}
& q_{3}(k)=\frac{d_{n}^{-2}}{d_{n}^{-2}+2 d_{n}^{-2} \mu_{n}}\left\{\frac{q_{2}(k)}{d_{n}^{-2}+2 d_{n}^{-2} \mu_{n}}-\mathbf{k}^{2}\right\} \\
& q_{4}(k)=\frac{q_{2}(k)}{d_{n}^{-2}+2 d_{n}^{-2} \mu_{n}}-\frac{2 d_{n}^{-2} \mu_{n} q_{1}(k)+d_{n}^{-2} \mathbf{k}^{2}}{d_{n}^{-2}+2 d_{n}^{-2} \mu_{n}}
\end{aligned}
$$

So the Fourier transform of $\mathbb{1}-Q_{n} \square^{-1} Q_{n}^{*}$ is

$$
\left[\begin{array}{cc}
\frac{2 d_{n}^{-2} \mu_{n}}{d_{n}^{-2}+2 d_{n}^{-2} \mu_{n}}+q_{3}(k)+O\left(\frac{\mu_{n}|k|^{2}}{d_{n}^{4}}\right)+O\left(\frac{|k|^{3}}{d_{n}^{2}}\right) & \frac{d_{n}^{-1} k_{0}}{d_{n}^{-2}+2 d_{n}^{-2} \mu_{n}}+O\left(\frac{|k|^{3}}{d_{n}}\right) \\
-\frac{d_{n}^{-1} k_{0}}{d_{n}^{-2}+2 d_{n}^{-2} \mu_{n}}+O\left(\frac{\mu_{n}|k|^{2}}{d_{n}^{3}}\right)+O\left(\frac{|k|^{3}}{d_{n}}\right) & q_{4}(k)+O\left(|k|^{3}\right)
\end{array}\right] .
$$

Unraveling the definitions and simplifying gives

$$
\begin{aligned}
& q_{3}(k)=\frac{d_{n}^{-2}}{\left(d_{n}^{-2}+2 d_{n}^{-2} \mu_{n}\right)^{2}}\left\{k_{0}^{2}+d_{n}^{-2} \mathbf{k}^{2}+2 \frac{\mu_{n}}{d_{n}^{2}} q_{1}(k)\right\} \\
& \left.q_{4}(k)=\frac{1}{d_{n}^{-2}+2 d_{n}^{-2} \mu_{n}}\left\{k_{0}^{2}+d_{n}^{-2} \mathbf{k}^{2}+2 \frac{\mu_{n}}{d_{n}^{2}} \mathbf{k}^{2}\right)\right\} .
\end{aligned}
$$


When $n$ is large, that is, deep in the 'elliptic regime,' the parameter $d_{n} \gg$ 1 and $d_{n}^{-2} \mu_{n}$ is essentially constant and the Fourier transform of $1-Q_{n} \square^{-1} Q_{n}^{*}$ is roughly, for small $k$

$$
\left[\begin{array}{cc}
1 & 0 \\
0 & \frac{k_{0}^{2}}{2 d_{n}^{-2} \mu_{n}}+\mathbf{k}^{2}
\end{array}\right]
$$

We see an elliptic operator in the tangential direction and a mass in the radial direction.

On the other hand, when $n$ is small, that is, early in the 'parabolic regime,' the parameter $d_{n}=1$ and $\mu_{n} \ll 1$ and the Fourier transform of $\mathbb{1}-Q_{n} \square^{-1} Q_{n}^{*}$ is roughly, for small $k$

$$
\left[\begin{array}{cc}
k_{0}^{2}+\mathbf{k}^{2} & k_{0} \\
-k_{0} & k_{0}^{2}+\mathbf{k}^{2}
\end{array}\right]
$$

The eigenvalues of this matrix are

$$
\pm i k_{0}+k_{0}^{2}+\mathbf{k}^{2} \approx \pm i k_{0}+\mathbf{k}^{2}
$$

which are parabolic operators.

A.4.c. Some Operators in Momentum Space. We here gather together some momentum space properties of the operators $\mathbb{D}_{n}$ and $\tilde{\mathbb{D}}_{n}$ that are used in the computations leading up to (A.15).

Lemma A.2. (a) If $p$ is bounded away from zero, then

$$
\hat{\mathbb{D}}_{n}^{-1}(p)=O\left(\left[\begin{array}{ll}
d_{n}^{-2} & d_{n}^{-1} \\
d_{n}^{-1} & 1
\end{array}\right]\right)
$$

(b) If $\ell \neq 0$, then

$$
\hat{\mathbb{D}}_{n}^{-1}(k+\ell) \hat{\mathbb{D}}_{n}(k)=O\left(\left[\begin{array}{cc}
d_{n}^{-2} \mu_{n}+|k| & d_{n}^{-1}|k| \\
d_{n}^{-1} \mu_{n}+d_{n}|k| & |k|
\end{array}\right]\right)
$$

(c)

$$
\widehat{\tilde{\mathbb{D}}}_{n}(k)=O\left(\left[\begin{array}{cc}
d_{n}^{-2} \mu_{n}+|k|^{2} & d_{n}^{-1}|k| \\
d_{n}^{-1}|k| & |k|^{2}
\end{array}\right]\right)
$$

(d) If $\ell \neq 0$, then

$$
\hat{\mathbb{D}}_{n}^{-1}(k+\ell) \widehat{\tilde{\mathbb{D}}}_{n}(k)=O\left(\left[\begin{array}{lr}
d_{n}^{-4} \mu_{n}+d_{n}^{-2}|k| & d_{n}^{-3}|k|+d_{n}^{-1}|k|^{2} \\
d_{n}^{-3} \mu_{n}+d_{n}^{-1}|k| & d_{n}^{-2}|k|+|k|^{2}
\end{array}\right]\right)
$$

Proof. (a) If $p$ is bounded away from zero, then

$$
\begin{aligned}
\hat{\mathbb{D}}_{n}^{-1}(p) & =\left[\begin{array}{cc}
2 \mu_{n}+\mathbf{p}^{2} & d_{n} p_{0} \\
-d_{n} p_{0} & \mathbf{p}^{2}
\end{array}\right]^{-1}=\frac{d_{n}^{-2}}{p_{0}^{2}+2 d_{n}^{-2} \mu_{n} \mathbf{p}^{2}+d_{n}^{-2} \mathbf{p}^{4}}\left[\begin{array}{cc}
\mathbf{p}^{2} & -d_{n} p_{0} \\
d_{n} p_{0} & 2 \mu_{n}+\mathbf{p}^{2}
\end{array}\right] \\
& =O\left(\left[\begin{array}{cc}
d_{n}^{-2} & d_{n}^{-1} \\
d_{n}^{-1} & 1
\end{array}\right]\right)
\end{aligned}
$$


(b) If $\ell \neq 0$, then $k+\ell$ is bounded uniformly away from zero and

$$
\begin{aligned}
\hat{\mathbb{D}}_{n}^{-1}(k+\ell) \hat{\mathbb{D}}_{n}(k) & =O\left(\left[\begin{array}{cc}
d_{n}^{-2} & d_{n}^{-1} \\
d_{n}^{-1} & 1
\end{array}\right]\right)\left[\begin{array}{cc}
2 \mu_{n}+\mathbf{k}^{2} & d_{n} k_{0} \\
-d_{n} k_{0} & \mathbf{k}^{2}
\end{array}\right] \\
& =O\left(\left[\begin{array}{cc}
d_{n}^{-2} \mu_{n}+|k| & d_{n}^{-1}|k| \\
d_{n}^{-1} \mu_{n}+d_{n}|k| & |k|
\end{array}\right]\right)
\end{aligned}
$$

(c) Using line 4 of (A.14.a),

$$
\begin{aligned}
& \widehat{\tilde{\mathbb{D}}}_{n}(k)=u_{n}(k)^{-2} \hat{\mathbb{D}}_{n}(k)\left\{u_{n}(k)^{2} \hat{\mathbb{D}}_{n}^{-1}(k) \widehat{\tilde{\mathbb{D}}}_{n}(k)\right\} \\
& =\left(1+O\left(|k|^{2}\right)\left[\begin{array}{cc}
2 \mu_{n}+\mathbf{k}^{2} & d_{n} k_{0} \\
-d_{n} k_{0} & \mathbf{k}^{2}
\end{array}\right]\right. \\
& {\left[\begin{array}{cc}
1+\frac{2 \mu_{n}}{u_{n}(k)^{2}}+\mathbf{k}^{2}+O\left(d_{n}^{-2} \mu_{n}|k|^{2}\right)+O\left(|k|^{3}\right) & \frac{d_{n} k_{0}}{u_{n}(k)^{2}}+O\left(d_{n}^{-1}|k|^{3}\right) \\
-d_{n} k_{0}+O\left(d_{n}^{-1} \mu_{n}|k|^{2}\right)+O\left(d_{n}|k|^{3}\right) & 1+\mathbf{k}^{2}+O\left(|k|^{3}\right)
\end{array}\right]^{-1} .}
\end{aligned}
$$

Next using (A.14.det)

$$
\begin{aligned}
& \widehat{\tilde{\mathbb{D}}}_{n}(k) \\
& =\left(1+O\left(|k|^{2}\right)\left[\begin{array}{cc}
2 \mu_{n}+\mathbf{k}^{2} & d_{n} k_{0} \\
-d_{n} k_{0} & \mathbf{k}^{2}
\end{array}\right] \frac{d_{n}^{-2}}{d_{n}^{-2}+2 d_{n}^{-2} \mu_{n}}\left\{1-\frac{q_{2}(k)}{d_{n}^{-2}+2 d_{n}^{-2} \mu_{n}}+O\left(|k|^{3}\right)\right\}\right. \\
& {\left[\begin{array}{cc}
1+\mathbf{k}^{2}+O\left(|k|^{3}\right) & -\frac{d_{n} k_{0}}{u_{n}(k)^{2}}+O\left(d_{n}^{-1}|k|^{3}\right) \\
d_{n} k_{0}+O\left(\frac{\mu_{n}|k|^{2}}{d_{n}}\right)+O\left(d_{n}|k|^{3}\right) & 1+\frac{2 \mu_{n}}{u_{n}(k)^{2}}+\mathbf{k}^{2}+O\left(\frac{\mu_{n}|k|^{2}}{d_{n}^{2}}\right)+O\left(|k|^{3}\right)
\end{array}\right]} \\
& =O(1) d_{n}^{-2}\left[\begin{array}{cc}
2 \mu_{n}+\mathbf{k}^{2} & d_{n} k_{0} \\
-d_{n} k_{0} & \mathbf{k}^{2}
\end{array}\right] \\
& {\left[\begin{array}{cc}
1+\mathbf{k}^{2}+O\left(|k|^{3}\right) & -\frac{d_{n} k_{0}}{u_{n}(k)^{2}}+O\left(d_{n}^{-1}|k|^{3}\right) \\
d_{n} k_{0}+O\left(\frac{\mu_{n}|k|^{2}}{d_{n}}\right)+O\left(d_{n}|k|^{3}\right) & 1+\frac{2 \mu_{n}}{u_{n}(k)^{2}}+\mathbf{k}^{2}+O\left(\frac{\mu_{n}|k|^{2}}{d_{n}^{2}}\right)+O\left(|k|^{3}\right)
\end{array}\right]} \\
& =O(1) d_{n}^{-2}\left[\begin{array}{cc}
2 \mu_{n}+O\left(d_{n}^{2}|k|^{2}+\mu_{n}|k|^{2}\right) & d_{n} k_{0}+O\left(d_{n}|k|^{3}+\frac{\mu_{n}}{d_{n}}|k|^{3}\right) \\
-d_{n} k_{0}+O\left(d_{n}^{-1} \mu_{n}|k|^{4}+d_{n}|k|^{4}\right) & O\left(d_{n}^{2}|k|^{2}+\mu_{n}|k|^{2}\right)
\end{array}\right] .
\end{aligned}
$$

So

$$
\widehat{\tilde{\mathbb{D}}}_{n}(k)=O\left(\left[\begin{array}{cc}
d_{n}^{-2} \mu_{n}+|k|^{2} & d_{n}^{-1}|k| \\
d_{n}^{-1}|k| & |k|^{2}
\end{array}\right]\right)
$$


(d) If $\ell \neq 0$, then

$$
\begin{aligned}
\hat{\mathbb{D}}_{n}^{-1}(k+\ell) \widehat{\tilde{\mathbb{D}}}_{n}(k) & =O\left(\left[\begin{array}{cc}
d_{n}^{-2} & d_{n}^{-1} \\
d_{n}^{-1} & 1
\end{array}\right]\left[\begin{array}{cc}
d_{n}^{-2} \mu_{n}+|k|^{2} & d_{n}^{-1}|k| \\
d_{n}^{-1}|k| & |k|^{2}
\end{array}\right]\right) \\
& =O\left(\left[\begin{array}{lc}
d_{n}^{-4} \mu_{n}+d_{n}^{-2}|k| & d_{n}^{-3}|k|+d_{n}^{-1}|k|^{2} \\
d_{n}^{-3} \mu_{n}+d_{n}^{-1}|k| & d_{n}^{-2}|k|+|k|^{2}
\end{array}\right]\right) .
\end{aligned}
$$

Open Access. This article is distributed under the terms of the Creative Commons Attribution 4.0 International License (http://creativecommons.org/licenses/by/4. $0 /$ ), which permits unrestricted use, distribution, and reproduction in any medium, provided you give appropriate credit to the original author(s) and the source, provide a link to the Creative Commons license, and indicate if changes were made.

\section{References}

[1] Abrikosov, A.A., Gorkov, L.P., Dzyaloshinski, I.E.: Methods of Quantum Field Theory in Statistical Physics. Dover Publications, New York (1963)

[2] Balaban, T.: A low temperature expansion for classical $N$-vector models. I. A renormalization group flow. Commun. Math. Phys. 167, 103-154 (1995)

[3] Balaban, T., Feldman, J., Knörrer, H., Trubowitz, E.: A functional integral representation for many boson systems. I: the partition function. Annales Henri Poincaré 9, 1229-1273 (2008)

[4] Balaban, T., Feldman, J., Knörrer, H., Trubowitz, E.: A functional integral representation for many boson systems. II: correlation functions. Annales Henri Poincaré 9, 1275-1307 (2008)

[5] Balaban, T., Feldman, J., Knörrer, H., Trubowitz, E.: Power series representations for complex bosonic effective actions. I. A small field renormalization group step. J. Math. Phys. 51, 053305 (2010)

[6] Balaban, T., Feldman, J., Knörrer, H., Trubowitz, E.: Power series representations for complex bosonic effective actions. II. A small field renormalization group flow. J. Math. Phys. 51, 053306 (2010)

[7] Balaban, T., Feldman, J., Knörrer, H., Trubowitz, E.: The temporal ultraviolet limit for complex bosonic many-body models. Annales Henri Poincaré 11, 151350 (2010)

[8] Balaban, T., Feldman, J., Knörrer, H., Trubowitz, E.: The temporal ultraviolet limit. In: Fröhlich, J., Salmhofer, M., De Roeck, W., Cugliandolo, L. (eds.) Quantum Theory from Small to Large Scales, Ecole de Physique des Houches, 2010, pp. 99-170. Oxford University Press, Oxford (2012)

[9] Balaban, T., Feldman, J., Knörrer, H., Trubowitz, E.: Bloch Theory for Periodic Block Spin Transformations. Preprint (2016). arXiv:1609.00964

[10] Balaban, T., Feldman, J., Knörrer, H., Trubowitz, E.: Operators for Parabolic Block Spin Transformations. Preprint (2016). arXiv:1609.00971

[11] Balaban, T., Feldman, J., Knörrer, H., Trubowitz, E.: Power Series Representations for Complex Bosonic Effective Actions. III. Substitution and Fixed Point Equations. Preprint (2016). arXiv:1609.00961 
[12] Balaban, T., Feldman, J., Knörrer, H., Trubowitz, E.: The Algebra of Block Spin Renormalization Group Transformations. Preprint (2016). arXiv:1609.00966

[13] Balaban, T., Feldman, J., Knörrer, H., Trubowitz, E.: The Small Field Parabolic Flow for Bosonic Many-Body Models: Part 1-Main Results and Algebra. Preprint (2016). arXiv:1609.01745

[14] Balaban, T., Feldman, J., Knörrer, H., Trubowitz, E.: The Small Field Parabolic Flow for Bosonic Many-Body Models: Part 2-Fluctuation Integral and Renormalization. Preprint (2016). arXiv:1609.01746

[15] Balaban, T., Feldman, J., Knörrer, H., Trubowitz, E.: The Small Field Parabolic Flow for Bosonic Many-Body Models: Part 3-Nonperturbatively Small Errors. Preprint (2016). arXiv:1609.01747

[16] Balaban, T., Feldman, J., Knörrer, H., Trubowitz, E.: The Small Field Parabolic Flow for Bosonic Many-Body Models: Part 4-Background and Critical Field Estimates. Preprint (2016). arXiv:1609.01748

[17] Benfatto, G.: Renormalization group approach to zero temperature Bose condensation. In: Rivasseau, V. (ed.) Constructive Physics. Springer Lecture Notes in Physics, vol. 446, pp. 219-247. Springer, Berlin (1995)

[18] Bogoliubov, N.N.: On the theory of superfluidity. J. Phys. (USSR) 11, 23-32 (1947)

[19] Brydges, D.C., Federbush, P.: The cluster expansion in statistical physics. Commun. Math. Phys. 49, 233-246 (1976)

[20] Brydges, D.C., Federbush, P.: The cluster expansion for potentials with exponential fall-off. Commun. Math. Phys. 53, 19-30 (1977)

[21] Cenatiempo, S., Giuliani, A.: Renormalization theory of a two dimensional Bose gas: quantum critical point and quasi-condensed state. J. Stat. Phys. 157, 755$829(2014)$

[22] Coleman, S.: Secret symmetry. An introduction to spontaneous symmetry breakdown and gauge fields. In: Zichichi, A. (ed.) Laws of Hadronic Matter, pp. 138215. Academic Press, New York/London (1975)

[23] Fetter, A.L., Walecka, J.D.: Quantum Theory of Many-Particle Systems. McGraw-Hill, New York (1971)

[24] Gawȩdzki, K., Kupiainen, A.: A rigorous block spin approach to massless lattice theories. Commun. Math. Phys. 77, 31-64 (1980)

[25] Kadanoff, L.P.: Scaling laws for Ising models near $T_{c}$. Physics 2, 263 (1966)

[26] Lieb, E.H., Seiringer, R., Solovej, J.P., Yngvason, J.: The Mathematics of the Bose Gas and Its Condensation. Birkhäuser, Basel (2005)

[27] Negele, J.W., Orland, H.: Quantum Many-Particle Systems. Addison-Wesley, Reading (1988)

[28] Pitaevskii, L., Stringari, S.: Bose-Einstein Condensation. Clarendon Press, Oxford (2003)

[29] Seiringer, R.: Cold quantum gases and Bose-Einstein condensation. In: Fröhlich, J., Salmhofer, M., De Roeck, W., Cugliandolo, L. (eds.) Quantum Theory from Small to Large Scales, Ecole de Physique des Houches, 2010, pp. 429-466. Oxford University Press, Oxford (2012)

[30] Weinberg, S.: The Quantum Theory of Fields, Volume II. Modern Applications. Cambridge Press, Cambridge (1998) 
Tadeusz Balaban

Department of Mathematics

Rutgers, The State University of New Jersey

110 Frelinghuysen Rd

Piscataway NJ08854-8019

USA

e-mail: tbalaban@math.rutgers.edu

Joel Feldman

Department of Mathematics

University of British Columbia

Vancouver BCV6T 1Z2

Canada

e-mail: feldman@math.ubc.ca

Horst Knörrer and Eugene Trubowitz

Mathematik

ETH-Zürich

ETH-Zentrum

8092 Zürich

Switzerland

e-mail: horst.knoerrer@math.ethz.ch

Eugene Trubowitz

e-mail: eugene.trubowitz@math.ethz.ch

Communicated by Vieri Mastropietro.

Received: January 20, 2017.

Accepted: March 30, 2017. 